\title{
Ecdysone coordinates plastic growth with robust pattern in the developing
}

\section{wing}

André Nogueira Alves1,2*, Marisa Mateus Oliveira1*, Takashi Koyama1,3,

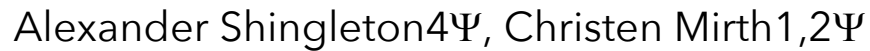

1 Instituto Gulbenkian de Ciência, Oeiras, Portugal

2 School of Biological Sciences, Monash University, Melbourne, Victoria, Australia

3 Department of Biology, University of Copenhagen, Copenhagen, Denmark

4 Department of Biological Sciences, University of Illinois at Chicago, Chicago, Illinois, USA

* These authors contributed equally to this work.

$\Psi$ Co-senior authors.

Keywords: phenotypic plasticity, developmental robustness, growth rates, patterning rates, Achaete, Senseless, wing disc, Drosophila melanogaster

Running title: Plastic Growth \& Robust Pattern 


\section{Abstract}

Animals develop in unpredictable, variable environments. In response to environmental change some aspects of development adjust to generate plastic phenotypes. Other aspects of development, however, are buffered against environmental change to produce robust phenotypes. How organ development is coordinated to accommodate both plastic and robust developmental responses is poorly understood. Here, we demonstrate that the steroid hormone ecdysone coordinates both plasticity of organ size and robustness of organ pattern in the developing wings of the fruit fly Drosophila melanogaster. Using fed and starved larvae that lack a prothoracic glands, which synthesise ecdysone, we show that nutrition regulates growth both via ecdysone and an ecdysone-independent mechanism, while nutrition regulates patterning only via ecdysone. We then demonstrate that growth shows a linear response to ecdysone concentration, while patterning shows a threshold response. Collectively, these data support a model where nutritionally-regulated ecdysone fluctuations confer plasticity by regulating disc growth as a graded response to basal ecdysone levels, and confers robustness by initiating patterning only once ecdysone peaks exceeds a threshold concentration. This could represent a generalizable mechanism through which hormones coordinate plastic growth with robust patterning in the face of environmental change.

\section{Introduction}

Developing animals respond to changes in their environment in a multitude of ways, for example, altering how long and how fast they grow, the time it takes them to mature, and their reproductive output $[1,2]$. Other aspects of their phenotype, however, must be unresponsive to environmental change to ensure that they function correctly regardless of environmental conditions. This presents a particular problem for morphological traits of developing animals. For any given trait, some aspects, such as final organ size, vary with changes in the environment, a phenomenon termed plasticity [3-7]. Other aspects, like patterning the cell types within an organ necessary for it to function, remain constant across environmental conditions, and are thus termed robust [6-9]. For many organs, growth and patterning occur at the same time during development, and may even be regulated by the same hormones [6]. How then do organs achieve plasticity in size while maintaining robustness of pattern?

If we want to extract general principles of how organisms regulate their development in variable environments, we need to understand how developmental processes unfold over time. Several recent studies that have applied systems approaches to development offer excellent examples, frequently employing methods to quantify how gene expression patterns change over time. These studies have used 
the dynamic changes in expression patterns to uncover the rules governing how insects build their segments $[10,11]$, how the gene regulatory network underlying segmentation evolves [12-17], how morphogen gradients scale across organs and bodies [18-23], how sensory organs are positioned within epithelia [24], and how somites and digits form in vertebrates [25-27]. The power of these approaches is that they provide a framework for understanding how genes interact within a network to generate pattern that can be applied across a variety of contexts.

The success of these studies is, in part, due to the fact that the gene regulatory networks underlying each of these processes have been well described in their respective developmental contexts. In contrast, the gene regulatory networks governing growth and patterning at later stages of development, even at later stages of embryonic development, are not as well resolved. If we further complicate this by comparing development across environmental conditions and even across traits, approaches that rely on understanding the configuration of gene regulatory networks become much more difficult to implement.

Nevertheless, we can still use the principle of comparing the dynamics of developmental processes across environments to gain useful insights into the relationship between plasticity and robustness. Many types of environmental conditions impact organ development to induce changes in body and organ size. Malnutrition or starvation reduces growth rates in all animals, resulting in smaller body and organ sizes [28-30]. Similarly, changing temperature can alter animal growth. In insect species, rearing animals in warmer conditions results in smaller adult body sizes when compared to animals reared under cooler conditions [31-38]. Other factors like oxygen availability and the presence of toxic or noxious compounds also act to alter animal sizes [39-41]. Examining how organ growth and patterning progress across these environmental conditions helps us to understand how these two processes are coordinated. 


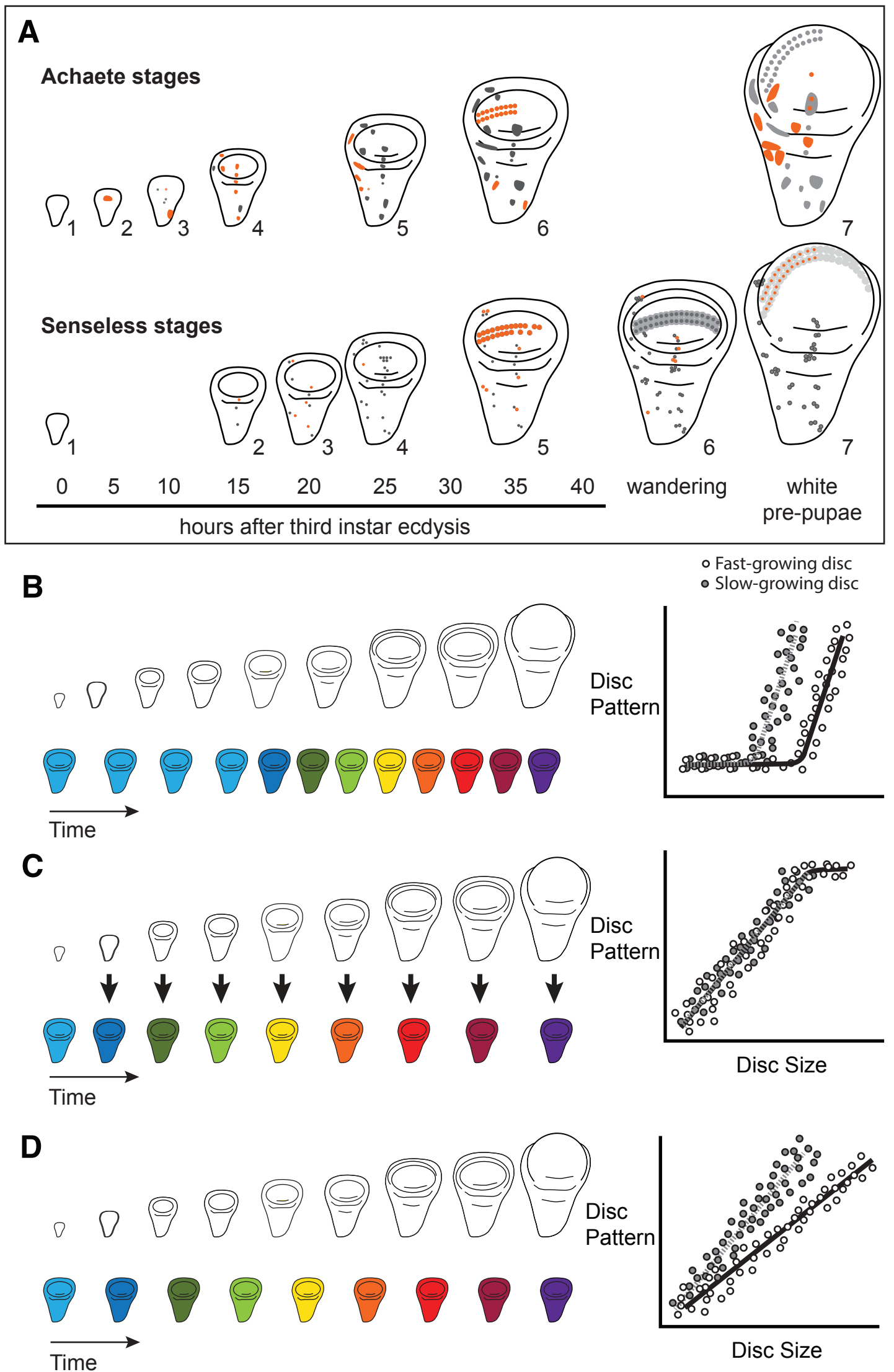


Figure 1: Quantitative assessments of the progression of patterning allow us to test hypotheses about the relationship between the size and patterning stage of the developing wing. A) The staging scheme developed by Oliveira et al. 2014 to quantify the progression of Achaete and Senseless pattern. The pattern elements shown in orange are diagnostic for each stage, which is indicated by the number beside the disc. (B-D) The relationship between wing disc size and patterning stage if; (B) Hypothesis 1: Wing discs grow first and then initiate pattern; (C) Hypothesis 2: Wing disc patterning is regulated by wing disc size (arrows); (D) Hypothesis 3: Wing disc pattern and growth are regulated at least partially independently.

We already have some understanding of the mechanisms that regulate growth and patterning in response to changing environmental conditions. The genetic mechanisms underlying plasticity in growth is best elucidated in insects. In insects, changes in available nutrition affect the synthesis and secretion of the conserved insulin-like peptides [42-44]. Insulin-like peptides bind to the Insulin Receptor in target tissues and activate the insulin signalling cascade, ultimately leading to increased growth $[43,45,46]$. Starvation reduces the concentration of insulin-like peptides in the bloodstream, and the resulting decrease in insulin signalling causes organs to grow more slowly $[44,47]$.

While changes in insulin signalling are known to affect organ size, they have little effect on organ pattern [48]. However, studies in the fruit fly Drosophila melanogaster have shown that, at least in this insect, insulin acts to control the synthesis of a second developmental hormone, the steroid hormone ecdysone [4952]. Most of the body and organ growth in $D$. melanogaster occurs in the third, and final, larval instar, after which the animal initiates metamorphosis at pupariation. Either starving or reducing insulin signalling early in the third instar delays the timing of ecdysone synthesis, thereby prolonging the length of the third instar and the time it takes to metamorphose [49-53]. Changing the rearing temperature also alters the timing of ecdysone synthesis early in larval development [54].

In addition to its effects on developmental time, ecdysone controls the growth of the developing adult organs [55-58]. In D. melanogaster larvae, many of the adult organs form and grow inside the larvae as pouches of cells called imaginal discs. If ecdysone synthesis is reduced or if the glands that produce ecdysone, the prothoracic glands (PG), are ablated, these imaginal discs grow at greatly reduced rates $[56,59]$.

Ecdysone signalling also regulates organ patterning. Reducing ecdysone signalling in either the wing imaginal disc or the developing ovary causes substantial delays in their patterning [56, 59-61]. In the wing disc, reducing ecdysone signalling stalls the progression of patterning of sensory bristles $[56,59]$. Similarly, in the ovary 
terminal filament cell specification and the rate of terminal filament addition both require ecdysone to progress normally $[60,61]$. Given its role in both the patterning and the growth of imaginal discs and ovaries, ecdysone is potentially a key coordinator of plastic growth and robust pattern.

Characterising organ growth rates is experimentally straight forward, requiring only accurate measurement of changes in organ size over time. To quantify the progression of organ patterning, however, requires developing a staging scheme. We previously developed such a scheme for the wing imaginal disc in $D$. melanogaster. This scheme makes use of the dynamic changes in expression from the moult to third instar to pupariation of up to seven patterning gene products in the developing wing [62]. Two of these patterning gene products, Achaete and Senseless, can be classed into seven different stages throughout third instar development ([62], Figure 1A), providing us with the ability to quantify the progression of wing disc pattern over a variety of conditions. In short, by describing patterning on a near continuous scale, our scheme not only allows us to determine under what conditions patterning is initiated, but also the rate at which it progresses.

The ability to simultaneously quantify both organ growth and pattern allows us to generate, and test, hypotheses regarding how ecdysone coordinates plastic growth with robust pattern. One hypothesis is that growth and patterning occur at different times, with ecdysone driving growth first then pattern later, or vice versa [6]. If this were true, we would expect to identify an interval where ecdysone concentrations primarily affected growth and a second interval where they affected mostly pattern (Figure 1B). There is some precedence for this idea; most of the patterning in the wing discs and ovaries of $D$. melanogaster occurs $15 \mathrm{~h}$ after the moult to the third larval instar [60]. Similarly, wing discs are known to grow faster in the early part of the third instar and slow their growth in the mid-to-late third instar [63]. As a second hypothesis, ecdysone could coordinate plastic growth with robust pattern if the impacts of ecdysone on one of these processes depended on its effects on the other. For example, morphogens are known to regulate both growth and patterning of the wing. If ecdysone controlled the action of morphogens we would expect the progression of patterning would be tightly coupled to growth over time, with different aspects of patterning being initiated at different disc sizes (Figure 1C). Finally, a third hypothesis is that ecdysone regulates growth and patterning of the wing discs independently, and that each process responds in a qualitatively and quantitively different manner to ecdysone [6]. As an example of this, we might see that growth rates increase in a graded response to increasing ecdysone while patterning shows threshold responses, or vice versa. If this were the case, we would 
expect that growth and the progression of pattern would be uncoupled over time (Figure 1D).

Here we test these hypotheses of whether and how ecdysone co-regulates plastic growth and robust pattern in wing imaginal discs in $D$. melanogaster. We blocked the production of ecdysone by genetically ablating the PG [56], and quantified the effects on growth and patterning rates throughout the third instar. We then manipulated the rate of ecdysone synthesis, by up-or down-regulating the activity of the insulin-signalling pathway in the PG $[52,64]$, to test how this alters the relationship between disc size and disc pattern. Finally, we tested our hypotheses about how a single steroid can regulate both plastic growth and robust patterning by conducting dose response experiments under two nutritional conditions. These studies provide a foundation for a broader understanding of how developmental hormones coordinate both plastic and robust responses across varying environmental conditions during animal development.

\section{Results}

\section{Ecdysone is necessary for the progression of growth and patterning}

To understand how ecdysone affects the dynamics of growth and patterning, we needed to be able to precisely manipulate ecdysone concentrations. For this reason, we made use of a technique we developed previously to genetically ablate the prothoracic glands (referred to as PGX) [56]. This technique pairs the temperature sensitive repressor of GAL4, GAL80 ${ }^{\text {ts }}$, with a prothoracic gland-specific GAL4 (phmGAL4) to drive an apoptosis-inducing gene (UAS-GRIM). GAL80 ts is active at $17^{\circ} \mathrm{C}$, where it represses GAL4 action, but inactive above $25^{\circ} \mathrm{C}$, which allows phm-GAL4 to drive expression of UAS-GRIM and ablate the PG $[65,66]$. Because ecdysone is required at every moult, we reared larvae from egg to third instar at $17^{\circ} \mathrm{C}$ to repress GAL4, then shifted the larvae to $29^{\circ} \mathrm{C}$ at the moult to the third instar to generate PGX larvae. This allows us to examine how eliminating ecdysone affects disc size and pattern in third instar wing imaginal discs, and to manipulate ecdysone concentrations by adding it back in specific concentrations to the food [56]. For simplicity, all the data from the two control strains (either the phm-GAL4; GAL80ts or UAS-GRIM parental strain crossed to $\left.\mathrm{w}^{1118}\right)$, were pooled in all analyses. 
Insect wing discs show damped exponential, or fast-then-slow, growth dynamics $[63,67]$, and have been modelled using a Gompertz function. The growth of wing discs from control and PGX larvae shows the same pattern, with a Gompertz function providing a significantly better fit to the relationship between log disc size and time than a linear function (ANOVA, linear vs. Gompertz model, $n>93, F>65$, $P<0.001$ for discs from both $P G X$ and control larvae). Growth of the discs, however, followed a significantly different trajectory in PGX versus control larvae (Figure 2). In control larvae, discs continue to grow until $42 \mathrm{~h}$ after ecdysis to the third instar (AEL3), when the larvae pupariate. In contrast, the wing imaginal discs of the PGX larvae stop growing at approximately $25 \mathrm{~h} \mathrm{AEL3}$, and at a significantly smaller size. This is despite the fact that PGX larvae do not pupariate, and so disc growth is not truncated by metamorphosis.

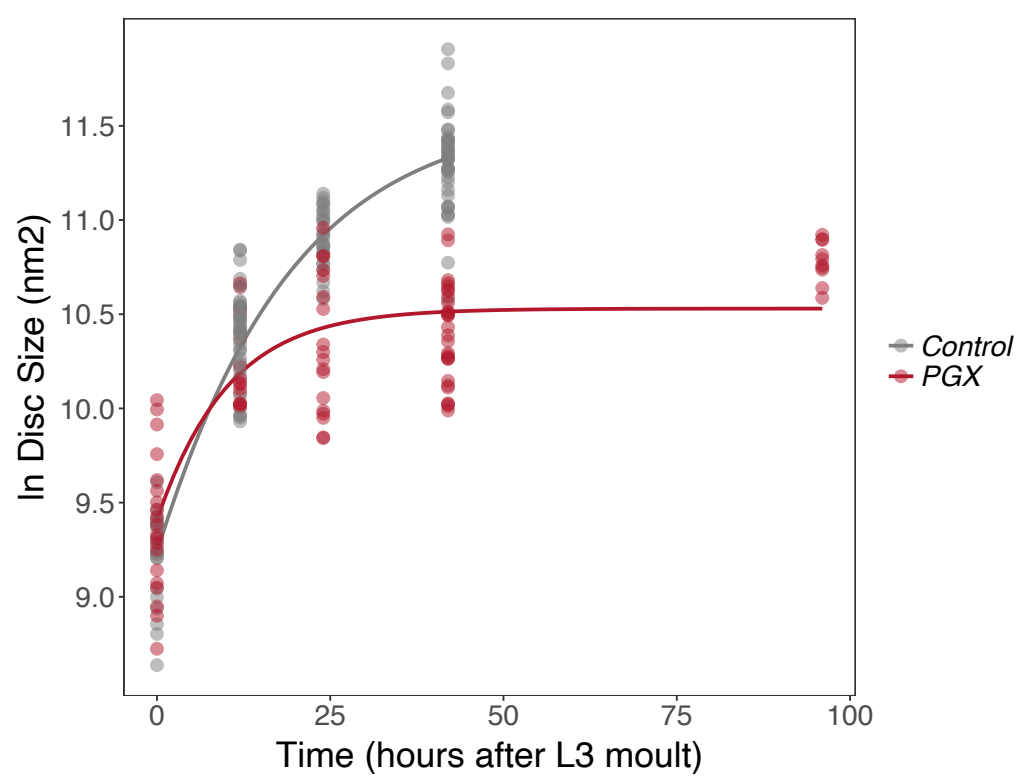

Figure 2: Growth rates of wing discs are reduced in larvae with genetically-ablated PG (PGX) versus control larvae. Curves are Gompertz functions of disc size against disc age (hours after ecdysis to the third larval instar, AEL3). Parameters for the curves are significantly different between PGX and control (Supplementary Table 1). Control genotypes are the pooled results from both parental controls (either the phm-GAL4; GAL80ts or UAS-GRIM parental strain crossed to $\mathrm{w}^{1118}$ ).

We next explored how loss of ecdysone affected the progression of wing patterning. We used the staging scheme that we previously devised in [62] to quantify the progression of wing disc patterning in PGX and control larvae. We selected two genes from this scheme, Achaete and Senseless, as they each progress 
through seven stages throughout the third instar. Further we can stain for both antigens in the same discs, which allowed us to compare disc size, Achaete stage, and Senseless stage in the same sample.

The progression of Achaete patterning was best fit by a Gompertz function for discs from both PGX and control larvae (ANOVA, linear v. Gompertz model, $n>48$, $F>10.4, P=0.002$ ) [62], and was significantly affected by loss of ecdysone. In control larvae, the wing discs progressed to Achaete stage 6 or 7 out of seven stages by $42 \mathrm{~h}$ AEL3, while in PGX larvae, discs of the same age had not passed Achaete stage 3, and had not matured past Achaete stage 5 by $92 \mathrm{~h}$ AEL3 (Figure 3A). The progression of Senseless patterning was best fit by a linear model, but again was significantly affected by loss of ecdysone. In control larvae, most discs had progressed to Senseless stage 6 out of seven stages by $42 \mathrm{~h}$ AEL3, while no disc progressed past Senseless stage 2 by $92 \mathrm{~h}$ AEL3 (Figure 3B, Supplementary Table 3).

We find no evidence of temporal separation between wing disc growth and the progression of pattern (compare Figures 2 and 3). Both growth and patterning progress at steady rates throughout most the third instar in control larvae, slowing down only at the later stages of development. The hypothesis that ecdysone coordinates plastic growth with robust pattern by acting on each process at different times (Figure 1C; Hypothesis 1) is therefore not correct.
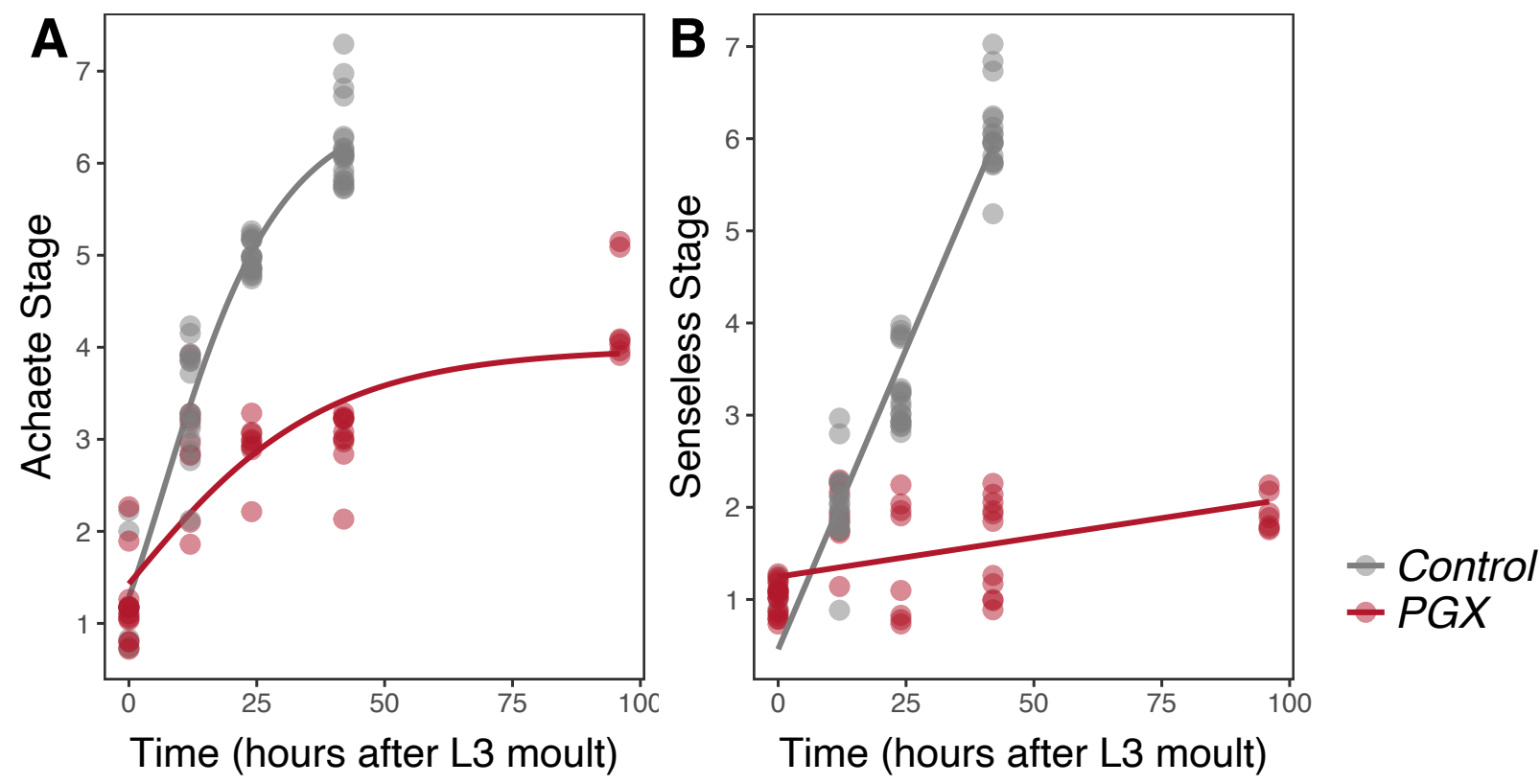

Figure 3: Achaete and Senseless patterning of wing discs is delayed in larvae with genetically-ablated PG (PGX) versus control larvae. (A) Curves are Gompertz functions of Achaete stage against time (hours AEL3). Parameters for the curves are significantly different between PGX and control (Supplementary Table 2). (B) Lines are linear regression of Senseless stage against time (hours AEL3). Parameters for the lines are significantly different 
between PGX and control (Supplementary Table 3). Control genotypes are the pooled results from both parental controls (either the phm-GAL4; GAL80ts or UAS-GRIM parental strain crossed to $\left.\mathrm{w}^{1118}\right)$.

To confirm that the lack of ecdysone was responsible for delayed patterning, and not a systemic response to the death of the glands, we performed a second experiment where we added either the active form of ecdysone (20hydroxyecdysone) or ethanol (the carrier) back to the food. PGX and control larvae were transferred onto either ecdysone or ethanol food and allowed to feed for $42 \mathrm{~h}$, after which we dissected their wing discs and examined their size and pattern. On the control (ethanol) food, wing discs from PGX larvae were smaller (Figure 4A) and showed reduced patterning for both Achaete (Figure 4B) and Senseless (Figure 4C) when compared to control genotypes. Adding $1 \mu \mathrm{g} / \mathrm{mg}$ food of ecdysone fully restored disc size, and Achaete and Senseless pattern, such that they were indistinguishable from control genotypes fed on ecdysone-treated food.

A

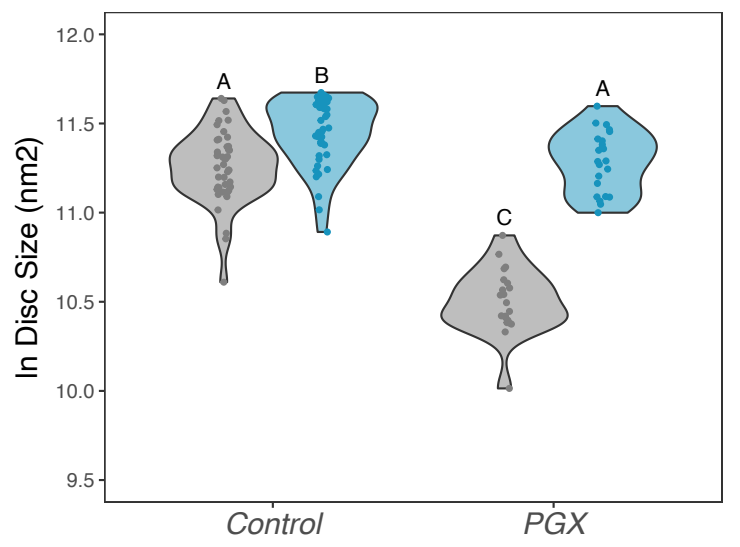
Ethanol Ecdysone

B

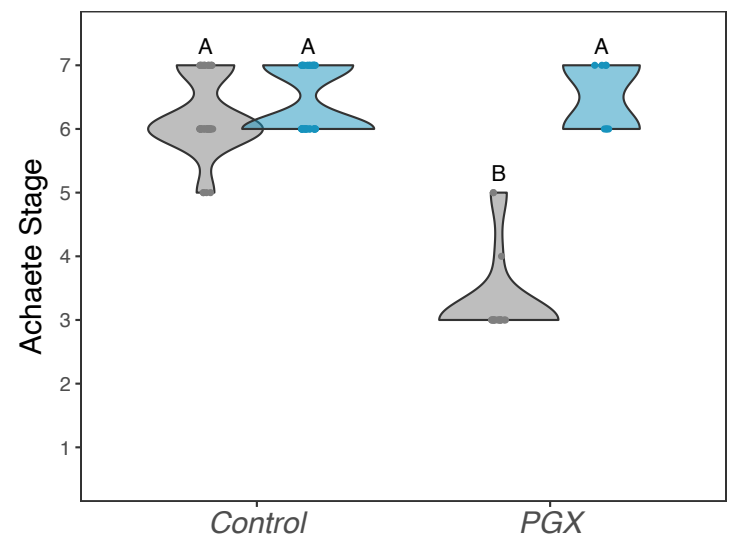

C

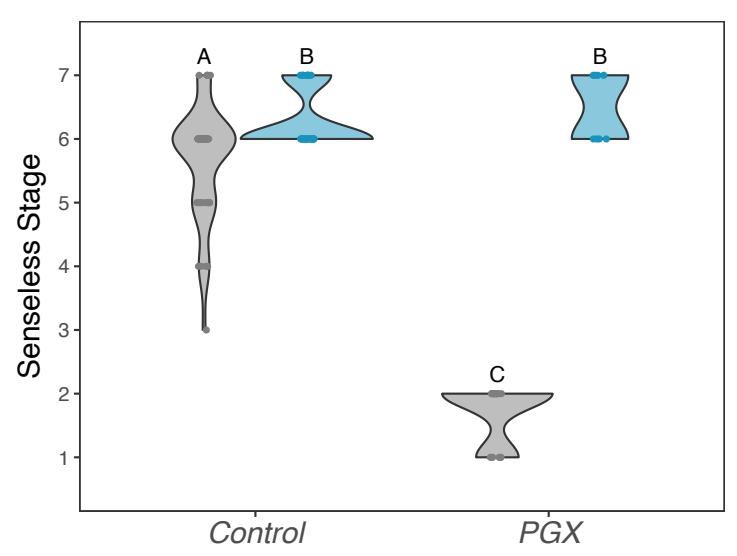

Figure 4: Supplementing PGX larvae with ecdysone rescues wing disc growth (A) and Achaete (B) and Senseless (C) patterning. Both the control and PGX larvae were exposed to ecdysone-treated food ( $0.15 \mathrm{mg}$ of 20-hydroxyecdysone) or ethanol-treated food (which 
contains the same volume of ethanol) at $0 \mathrm{~h}$ AL3E. Wing discs were removed at $42 \mathrm{~h} \mathrm{AL3E}$. Control genotypes are the pooled results from both parental controls (either the phm-GAL4; GAL80ts or UAS-GRIM parental strain crossed to $\mathrm{w}^{1118}$ ).

Collectively, these data indicate that ecdysone is necessary for the normal progression of growth and patterning in wing imaginal discs. The loss of ecdysone has a more potent effect on patterning, however, which is effectively shut-down in PGX larvae, than on disc growth, which continues more-or-less at a normal rate for the first 24 hours of the third instar in PGX larvae.

\section{Ecdysone rescues patterning and some growth in wing discs of yeast-starved larvae}

The observation that ecdysone is necessary to drive both normal growth and patterning suggests that it may play a role in coordinating growth and patterning across environmental conditions. However, to do so it must lie downstream of the physiological mechanisms that sense and respond to environmental change. As discussed above, ecdysone synthesis is regulated by the activity of the insulinsignalling pathway, which is in turn regulated by nutrition. Starving larvae of yeast early in the third instar both suppresses insulin-signalling and inhibits growth and patterning of organs $[59,60]$. We explored whether ecdysone was able to rescue some of this inhibition by transferring larvae immediately after the moult to $1 \%$ sucrose food that contained either ecdysone or ethanol and comparing their growth and patterning after 24 hours to wing discs from larvae fed on normal food. Both the PGX and control genotype failed to grow and pattern on the $1 \%$ sucrose with ethanol (Figure 5A-C). Adding ecdysone to the 1\% sucrose food rescued Achaete and Senseless patterning in both the control and the PGX larvae to levels seen in fed controls (Figure 5B, C). Ecdysone also partially rescued disc growth in PGX larvae, although not to the levels of the fed controls (Figure 5A). Collectively, these data suggest that the effect of nutrition on growth and patterning is at least partially mediated through ecdysone.

An important aspect of these data is that in PGX larvae, either supplementing the $1 \%$ sucrose food with ecdysone or feeding them on normal food both rescued wing disc growth (Figure 5A), albeit incompletely. This suggests that nutrition can drive growth through mechanisms independent of ecdysone, and vice versa. In contrast, nutrition alone only marginally promoted Achaete and Senseless patterning in starved PGX larvae, while ecdysone alone completely restored patterning. Further, 
even early patterning does not progress in PGX larvae (Figure 3). Thus, the effect of nutrition on patterning appears to be wholly mediated by ecdysone, while the effect of nutrition on growth appears to be partially mediated by ecdysone and partially through another independent mechanism. Ecdysone-independent growth appears to occur early in the third larval instar, however, since disc growth in PGX and control larvae is more-or-less the same in the first 12 hours after ecdysis to L3 (Figure 2).
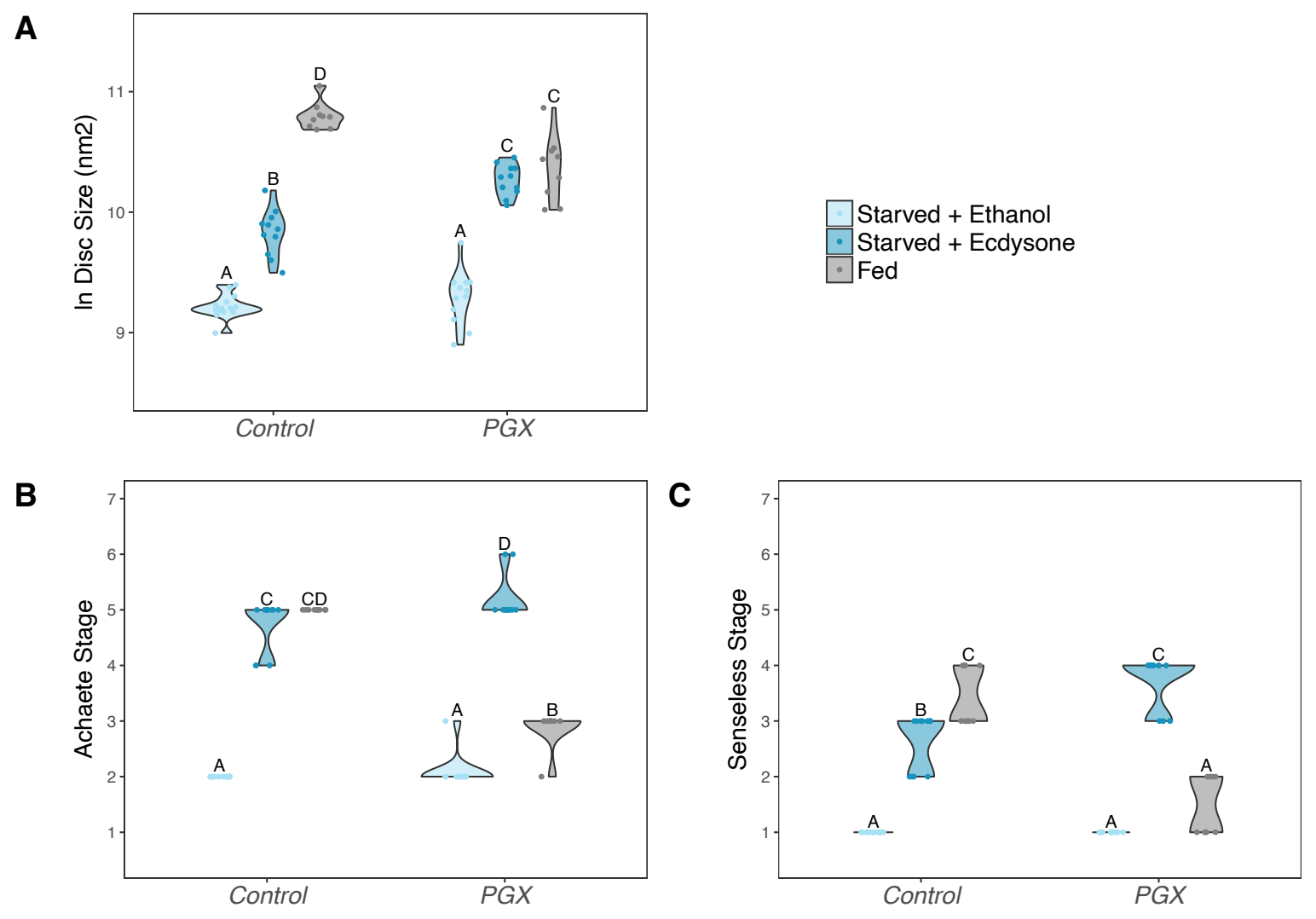

Figure 5: Supplementing PGX larvae with ecdysone is able to at least partially rescue the effect of yeast starvation on (A) wing discs growth, all fully rescue (B) Achaete, and (C) Senseless patterning. Both the control and PGX larvae were exposed from $0 \mathrm{~h} \mathrm{AL3E}$ to one of three food types: 1 ) starved + ecdysone - starvation medium containing $1 \%$ sucrose and $1 \%$ agar laced with ecdysone (0.15 mg of 20-hydroxyecdysone), 2) starved + ethanol - starvation medium treated with the same volume of ethanol, or 3) fed - normal fly food. Wing discs were removed at $24 \mathrm{~h}$ AL3E. Control genotypes are the pooled results from both parental controls (either the phm-GAL4; GAL80ts or UAS-GRIM parental strain crossed to $\mathrm{w}^{1118}$ ). Treatments marked with different letters are significantly different (Tukey HSD, $P<0.05$ ). 


\section{Ecdysone drives growth and patterning independently}

The data above suggest a model of growth and patterning, where both ecdysone and nutrition can drive growth, but where patterning is driven by ecdysone. We next focused on exploring how ecdysone regulates both growth and patterning. Patterning genes, particularly morphogens, are known to regulate growth, so one hypothesis is that ecdysone promotes patterning, which in turn promotes the ecdysone-driven component of disc growth. A second related hypothesis is that ecdysone-driven growth is necessary to promote patterning. Under either of these hypotheses, because the mechanisms regulating patterning and growth are interdependent, we would expect that changes in ecdysone levels would not change the relationship between disc size and disc pattern. An alternative hypothesis, therefore, is that ecdysone promotes growth and patterning through at least partially independent mechanisms. Under this hypothesis the relationship between size and patterning may change at different levels of ecdysone.

To distinguish between these two hypotheses, we increased or decreased the activity of the insulin-signalling pathway in the $P G$, which is known to increase or decrease the level of circulating ecdysone, respectively $[49,52,64,68]$. We then looked at how these manipulations affected the relationship between disc size and disc pattern, again focusing on Achaete and Senseless patterning. We increased insulin signalling in the PG by overexpressing $\ln R(p h m>\ln R)$, and reduced insulin signalling by overexpressing the negative regulator of insulin signalling PTEN (P0206>PTEN).

We found that a linear model is sufficient to capture the relationship between disc size and Achaete stage when we either increase $\left(p h m>I n R\right.$ : $A I C_{\text {linear }}-A_{1} C_{\text {logistic }}=$ 22 , ANOVA, $\left.F_{(25,27)}=1.71, P=0.2018\right)$ or decrease ecdysone synthesis rates (P0206>PTEN). Changing ecdysone levels, however, significantly changed the parameters of the linear model and altered the relationship between disc size and Achaete pattern. Specifically, increasing ecdysone level shifted the relationship so that later stages of Achaete patterning occurred in smaller discs (Figure 6B, Supplementary Table 5).

The relationship between Senseless pattern and disc size is best fit using a four-parameter logistic (threshold) function, which provides a significantly better fit to the data than a linear function $\left(\mathrm{AlC}_{\text {linear }}-\mathrm{AlC}_{\text {logistic }}=32.2 ; \mathrm{ANOVA}, F_{(44,46)}=25.8, P<\right.$ $0.001)$. Changing ecdysone levels significantly changed the parameters of the logistic model and altered the relationship between disc size and Senseless pattern (Figure 6A, Supplementary Table 4). Again, increasing ecdysone level shifted the relationship so that later stages of Senseless patterning occurred in smaller discs. Collectively, 
these data support the hypothesis that ecdysone acts on growth and patterning at least partially independently, and that patterning is not regulated by wing disc size (Figure 1D; Hypothesis 2).
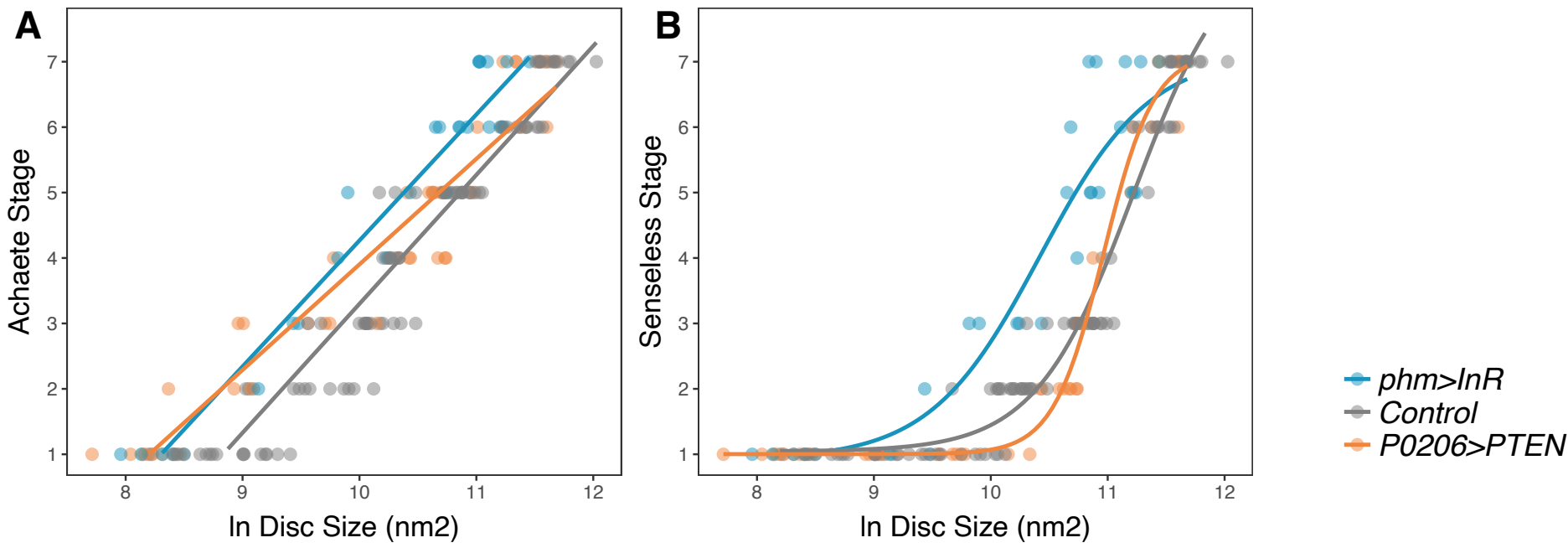

Figure 6: Changing level of ecdysteroidgenesis change the relationship between disc pattern and disc size. (A) The relationship between Senseless stage and disc size was fitted with a four-parameter logistic regression, the parameters of which are significantly different between genotypes (Supplementary Table 4). (B) The relationship between Achaete stage and disc size was fitted with a linear regression, the parameters of which are significantly different between genotypes (Supplementary Table 5).

\section{Ecdysone regulates disc growth and disc patterning through different mechanisms}

The data above support a model whereby environmental signals act through ecdysone to co-regulate growth and patterning, generating organs of variable size but invariable pattern. Further, growth is also regulated by an ecdysone independent mechanism, enabling similar progressions of pattern across discs of different sizes. An added nuance, however, is that ecdysone levels are not constant throughout development. Rather, the ecdysone titre fluctuates through a series of peaks throughout the third larval instar and the dynamics of these fluctuations are environmentally sensitive [69]. To gain further insight into how ecdysone co-regulates plasticity and robustness, we therefore explored which aspects of ecdysone dynamics regulate growth and patterning.

Two characteristics of ecdysone fluctuations appear to be important with respect to growth and patterning. First, the timing of the ecdysone peaks set the pace 
of development, initiating key developmental transitions such as larval wandering and pupariation $[52,64,69,70]$. Second, the basal levels of ecdysone appear to regulate the rate of body growth, with an increase in basal level leading to a reduction in body growth $[49,56,64,68,71]$. While several studies, including this one, have established that disc growth is positively regulated by ecdysone [56, 58, 72], whether disc growth is driven by basal levels or peaks of ecdysone is unknown.

There are a number of hypotheses as to how ecdysone levels may drive patterning and growth. One hypothesis is that patterning and ecdysone-regulated disc growth show a single threshold response, being initiated once ecdysone rises above a certain level. Alternatively, both may show a graded response, progressing at a rate that correlates with the level of circulating ecdysone. A more nuanced hypothesis is that patterning and growth are driven by sequentially increasing response thresholds to ecdysone. Under this hypothesis, discs would pattern and grow to a specific stage/disc size and not continue until ecdysone levels rose above the next threshold. Finally, disc patterning may show one type of response to ecdysone, while disc growth may show another. Separating these hypotheses requires both a pattern staging scheme (Figure 1) and the ability to titrate levels of circulating ecdysone.

To separate these hypotheses, we reared PGX larvae on standard food supplemented with a range of ecdysone concentrations $(0,6.25,12.5,25,50$ and 100 ng of ecdysone/mg of food). However, as noted above, disc growth early in the third larval instar is only moderately affected by ablation of the PG, potentially obfuscating the effects of supplemental ecdysone. In contrast, discs from starved PGX larvae show no growth or patterning without supplemental ecdysone. We therefore also reared PGX larvae on $20 \%$ sucrose / $1 \%$ agar medium (from here on referred to as 'starved' larvae) supplemented with a range of ecdysone concentrations. For both control genotypes and PGX larvae, increasing the concentration of ecdysone in the food increases the hemolymph titres of ecdysone (Supplementary Fig 1). We dissected discs at $5 \mathrm{~h}$ intervals starting immediately after the moult to the third instar ( $0 \mathrm{~h} \mathrm{AL3E}$ ) to $20 \mathrm{~h}$ AL3E. Because male and female larvae show differences in wing disc growth [73], we separated the sexes in this experiment and focused our analysis on female wing discs. 


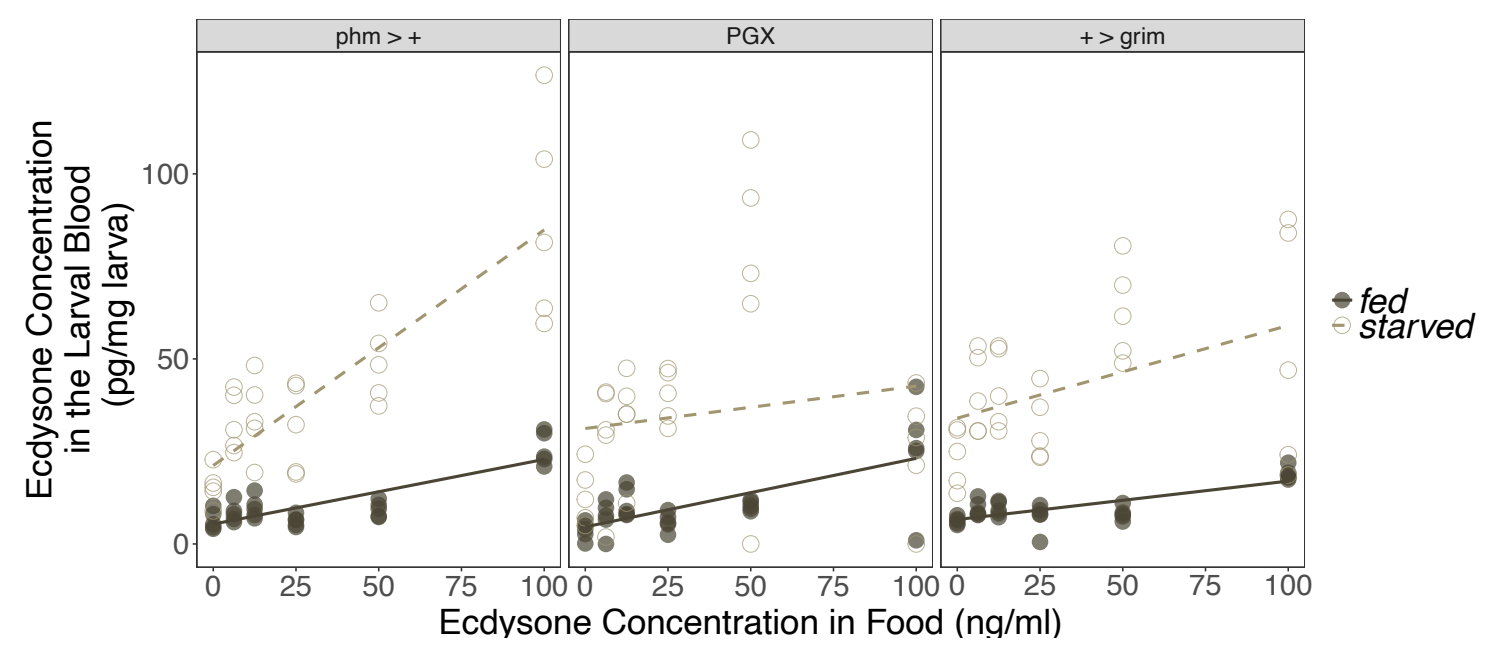

Supplementary Figure 1: The effect of $20 \mathrm{E}$ concentration in the food in larvae reared on either a regular diet (fed) or $20 \%$ sucrose / $1 \%$ agar diet (starved) on circulating ecdysone levels. There is a significant positive relationship between ecdysone concentration in the food and the concentration of ecdysone in the larval blood. Furthermore, starved larvae had higher ecdysone concentrations in their hemolymph than fed larvae.

As before, in both PGX and control larvae, wing disc growth was suppressed by starvation (Supplementary Figure 2). An increase in the concentration of supplemental ecdysone increased the rate of disc growth in starved PGX larvae such that there was a positive relationship between ecdysone concentration and the (linear) rate of disc growth (Figure 7, Supplementary Table 6 and 7). Thus, disc growth appears to show a graded response to ecdysone level in the absence of nutrition. In contrast, increasing ecdysone concentrations had no effect on disc growth rate in fed PGX larvae (Supplementary Figure 2, Supplementary Table 6). That is, the effect of nutrition on growth obfuscated the effect of ecdysone. This is not surprising since ablation of the PG has little effect on disc growth in the first 12 hours of the third instar in fed larvae (Figure 2), and again supports the hypothesis that disc growth early in the third instar is largely regulated by nutrition and not ecdysone [63]. 

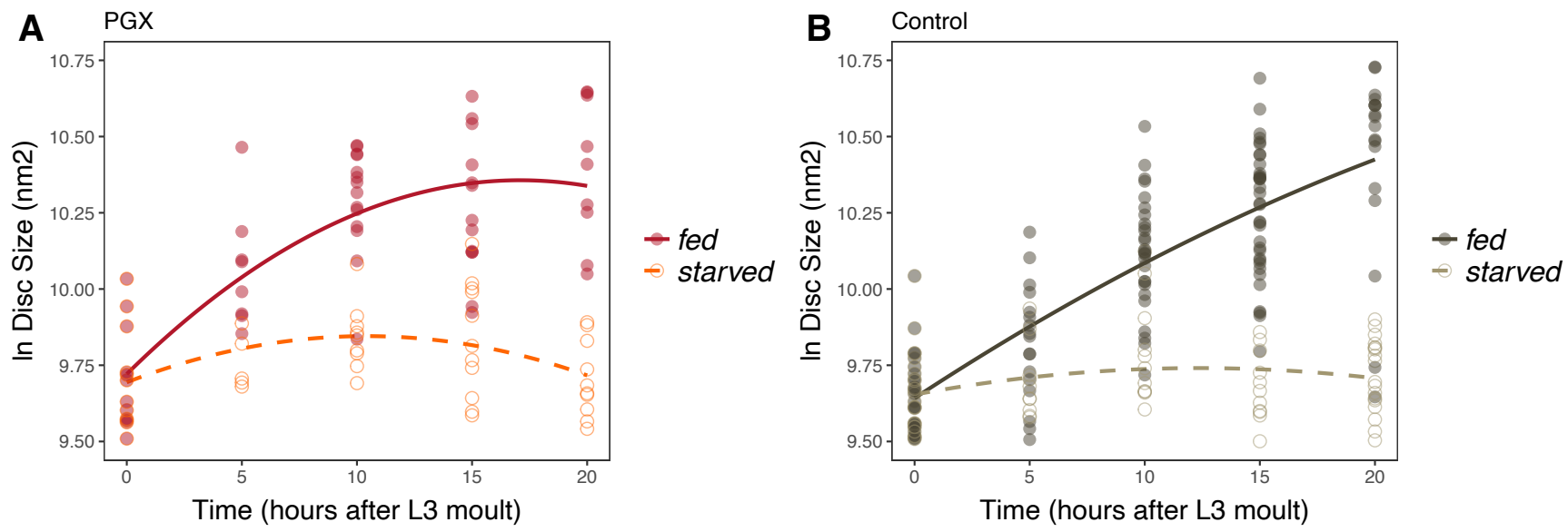

Supplementary Figure 2: Wing imaginal disc growth is suppressed in fed PGX larvae relative to controls, and in starved larvae of both genotypes. Wing disc growth was modelled as a quadratic, and there was a significant interaction between genotype (PGX v. Control) and nutrition (fed v. starved) on growth (Supplementary Table 7). Solid line/closed point = fed larvae, broken line/open point = starved larvae.

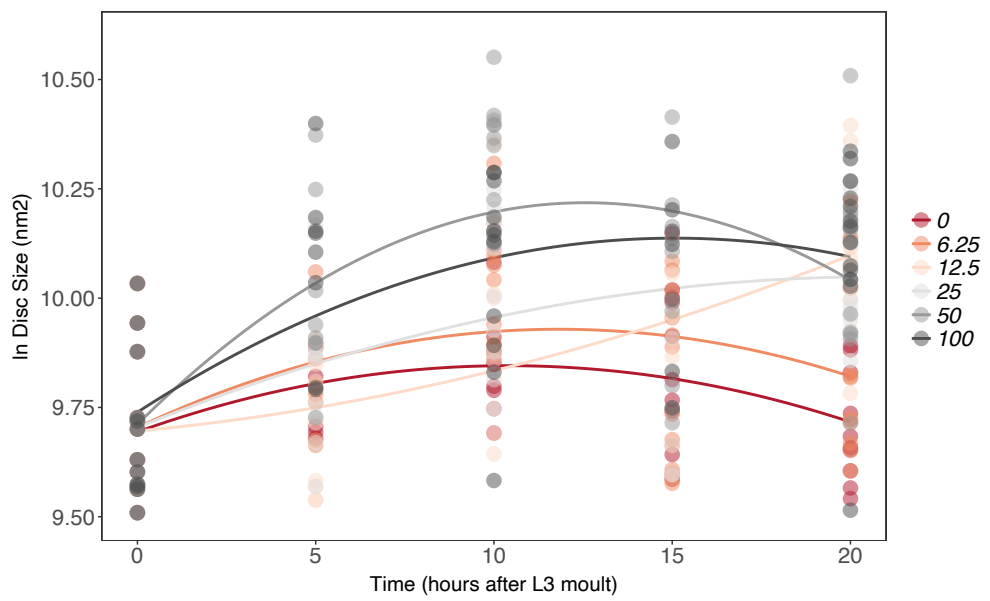

Figure 7: Effect of supplemental 20E on growth of the wing imaginal disc in starved PGX larvae. Growth was modelled as $S=E+T+T^{2}+E^{\star} T+E^{\star} T^{2}$, where $S=$ disc size, $E=20 \mathrm{E}$ concentration, and $T=$ disc age. There was a significant effect of $E$ on the linear growth rate of the wing imaginal discs (Supplementary Table 8 and 9). 


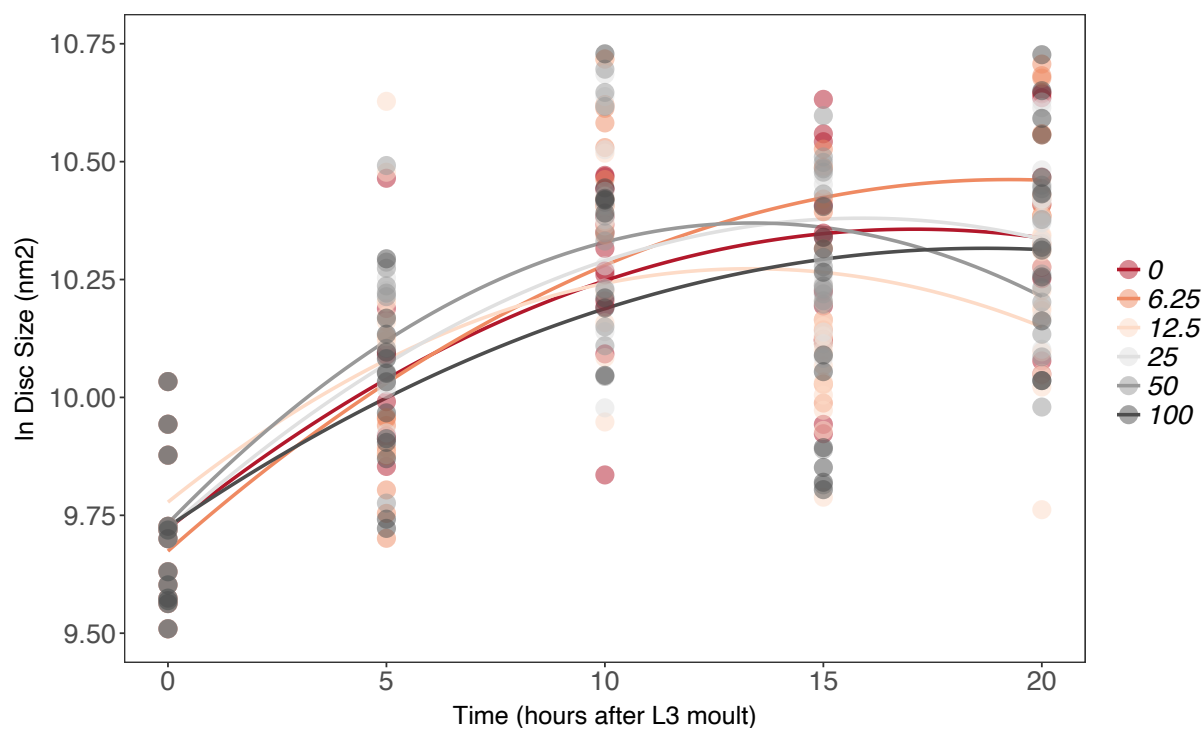

Supplementary Figure 3: There is no effect of supplemental 20E on growth of the wing imaginal disc in fed PGX larvae. Growth was modelled as $S=E+T+T^{2}+E^{\star} T+E^{\star} T^{2}$, where $S=$ disc size, $E=20 \mathrm{E}$ concentration, and $T=$ disc age. There was no significant effect of $E$ on the linear or quadratic growth rate of the wing imaginal discs (Supplementary Table 8).

The effect of ecdysone concentration on Achaete patterning was qualitatively different than its effect on growth. As before, Achaete patterning did not progress in either starved or fed PGX larvae (Supplementary Figure 4A). In contrast, Achaete patterning did progress in PGX larvae supplemented with $20 \mathrm{E}$. In order to explore how patterning progressed at different levels of $20 \mathrm{E}$, we again modelled the progression of Achaete patterning using a Gompertz function, fitting a linear function when the Gompertz function failed to fit (when patterning did not progress sufficiently to fit a Gompertz function). In both fed and starved females, $6.25 \mathrm{ng} \mathrm{ml}^{-1}$ ecdysone was insufficient to rescue patterning, discs from larvae fed $12.5 \mathrm{ng} \mathrm{ml}^{-1}$ ecdysone showed intermediate levels of patterning, while levels of ecdysone above $25 \mathrm{ng} \mathrm{m}^{-1}$ all rescued Achaete patterning to the same extent (Figure 8, Supplementary Table 8 and 9). Thus, unlike growth, Achaete patterning appears to show a threshold response to ecdysone level, not initiating unless ecdysone is above a certain level (12.5-25ng $\mathrm{ml}^{-1}$ ), but progressing at the same rate and to the same extent regardless of how high ecdysone is above this level. Comparing the timing of Achaete patterning in ecdysone-supplemented PGX larvae versus fed controls provides some indication of when in normal development this threshold is reached: Discs from fed control larvae began to reach Achaete stage 4 by $15 \mathrm{~h}$ AEL3 (Supplementary Figure 4), while discs from both fed and starved PGX larvae supplemented with $>25 \mathrm{ng} \mathrm{m}^{-1}$ of ecdysone began to reach stage 4 by $10 \mathrm{~h} \mathrm{AEL3}$ 
(Figure 8). This suggests that in control larvae, ecdysone levels sufficient to initiate Achaete patterning are only reached toward the middle of the third larval instar.
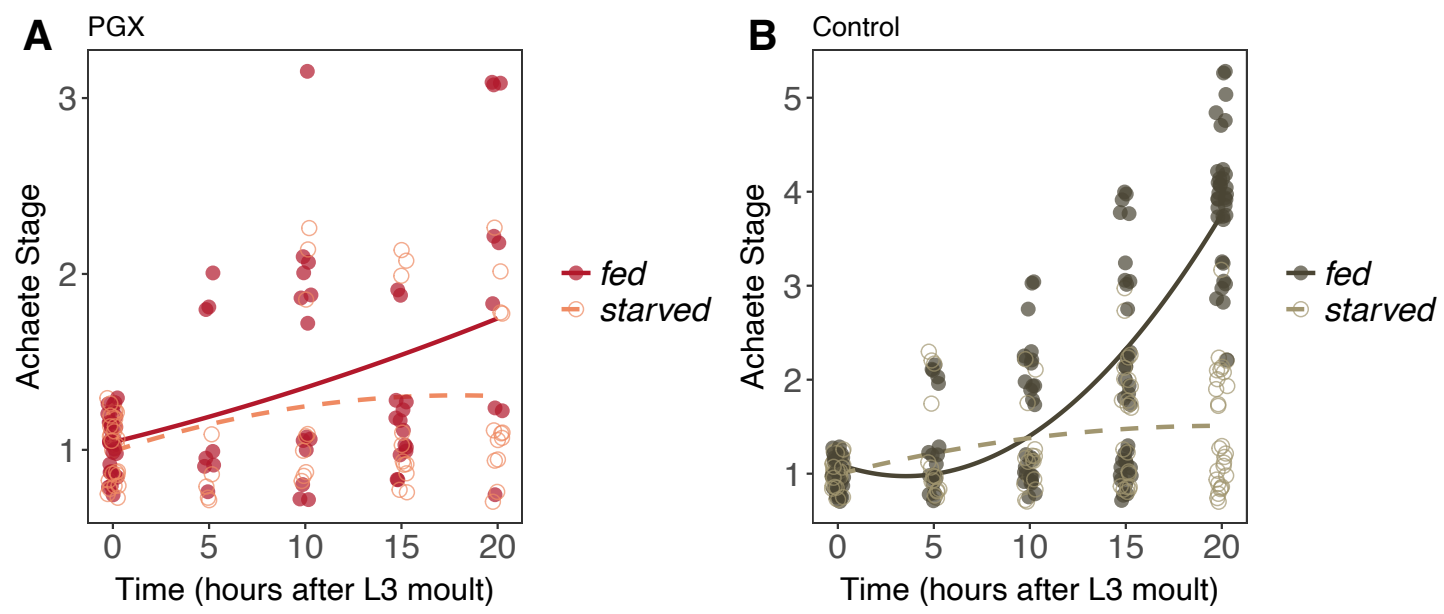

Supplementary Figure 4: Achaete patterning in wing discs from fed and starved PGX and control larvae. (A) Patterning does not progress in either fed or starved PGX larvae. (B) Patterning does not progress in starved control larvae but does in fed control larvae. There is a significant interaction between the effects of disc age and food on Achaete patterning in control larvae (orthogonal polynomial regression: $\mathrm{F}_{\text {food } * \text { disc } \text { age }^{2}=67.98, \mathrm{P}<}$ 0.001 ), but not in $P G X$ larvae (orthogonal polynomial regression: $F_{\text {food } * \text { disc age }}{ }^{2}=1.81, P=$ 0.163). See supplementary materials for details.
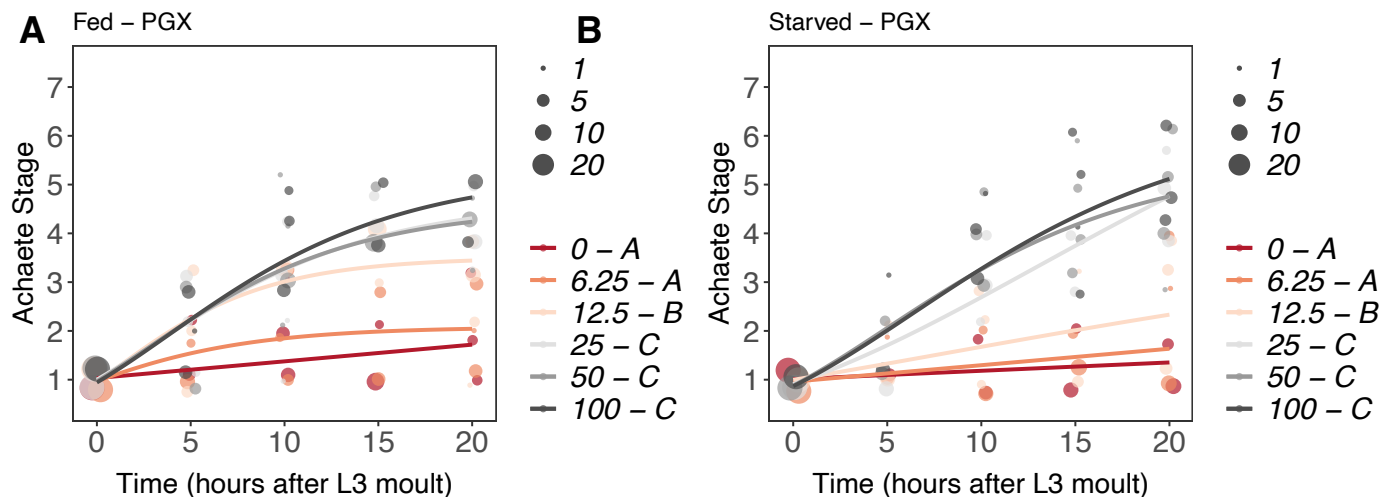

Figure 8: Effect of supplemental 20E on Achaete patterning of the wing imaginal disc in (A) fed and (B) starved PGX larvae. Growth is modelled either as a Gompertz or a linear function. Lines with different letters are significantly different. See supplementary tables 10 and 11 for statistical details.

Senseless patterning showed a similar threshold response to increases in ecdysone, although it did not progress as far as Achaete patterning, only achieving an average of stage 3 or stage 4 by $20 \mathrm{~h}$ AEL3 in fed and starved larvae, respectively. 
We again fit a linear model between disc age and Senseless pattern. As before, Senseless patterning did not progress in either starved or fed PGX larvae (Supplementary Figure 5). In both fed and starved females, supplemental ecdysone at or below $12.5 \mathrm{ng} \mathrm{ml}^{-1}$ was insufficient to rescue patterning, while supplemental ecdysone at or above $25 \mathrm{ng} \mathrm{m}^{-1}$ rescued patterning to the same extent (Figure 9). As for Achaete patterning, supplemental ecdysone initiated Senseless patterning in PGX larvae early versus fed controls. Discs from fed control larvae began to reach Senseless stage 3 at 20h AEL3 (Supplementary Figure 5), while discs from both fed and starved PGX larvae supplemented with $>25 \mathrm{ng} \mathrm{ml}^{-1}$ of ecdysone were at stage 3 by 10 h AEL3 (Figure 9).

Collectively, these data support a model of disc growth and patterning where ecdysone regulates disc growth as a graded response to basal levels of ecdysone, while ecdysone regulates disc patterning as a single threshold response.
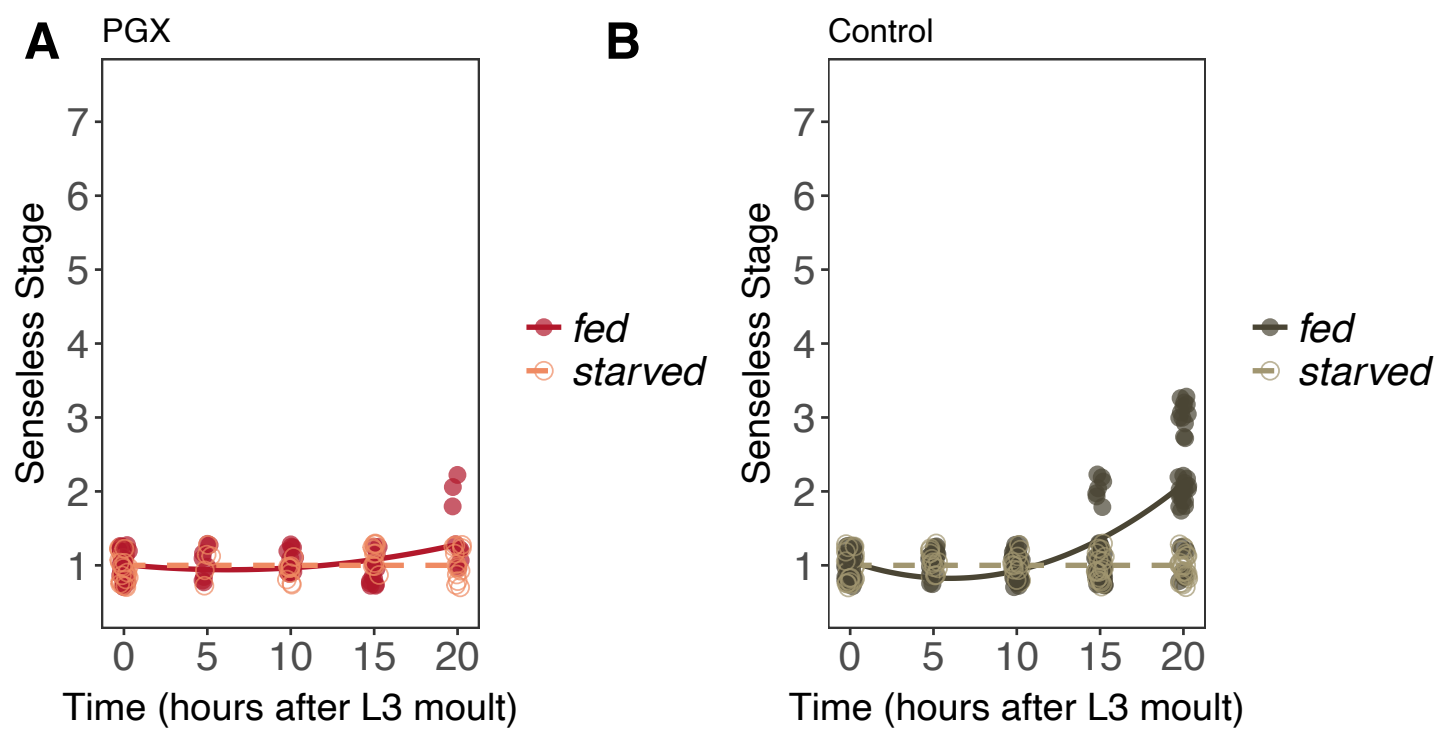

Supplementary Figure 5: Senseless patterning in wing discs from fed and starved PGX and control larvae. (A) Patterning does not progress in either fed or starved PGX larvae. (B) Patterning does not progress in starved control larvae but does in fed control larvae. There is a significant interaction between the effects of time and food on Achaete patterning in control larvae (linear regression: $F_{\text {food } * \text { time }}=67.98, P<0.001$ ). In PGX larvae Senseless patterning does not progress at all in starved larvae (linear regression: $F_{\text {time }}=0.057, P=0.82$ ), but does in fed larvae (linear regression: $F_{\text {time }}=9.76, P<0.01$ ). Control genotypes are the pooled results from both parental controls (either the phm-GAL4; GAL80ts or UAS-GRIM parental strain crossed to $\left.w^{1118}\right)$. 

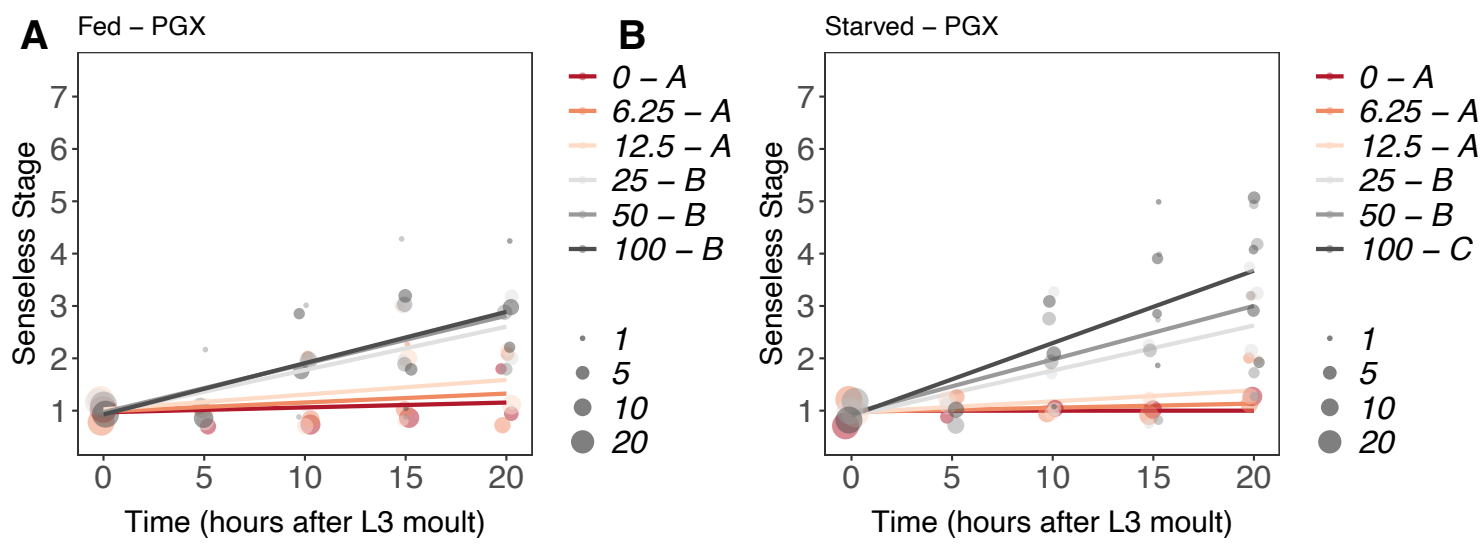

Figure 9: Effect of supplemental 20E on Senseless patterning of the wing imaginal disc in (A) fed and (B) starved PGX larvae. Growth is modelled as a linear function. See supplementary tables 12 and 13 for statistical details.

\section{Discussion}

Organs are remarkably good at achieving correct pattern across a broad range of environmental conditions that generate variation in size. While this might seem a simple feat when growth and patterning occur at separate times or are regulated by different hormones, it is considerably less simple if both growth and patterning occur at the same time and are regulated by the same endocrine signal. In this work, we explored how the wing discs of developing $D$. melanogaster use the same hormonal signal to coordinate both their growth and their progression of pattern. We found that ecdysone simultaneously regulates the plastic growth and robust patterning of the wing disc through independent mechanisms: plastic growth responds to ecdysone with a graded response, while robust patterning responds with a single threshold response. We propose that these differences in response represent a potentially general mechanism through which high levels of variation in one organ characteristic, for example size, could be coordinated with low levels variation in another characteristic of the same organ, for example pattern.

These data make an important contribution to our understanding of how environmental factors, specifically nutrition, affect growth and patterning in developing organs in Drosophila. During normal development, circulating ecdysone levels are low during the first 8 hours of the third larval instar, until attainment of a critical size initiates a hormonal cascade that causes ecdysone to fluctuate through a series of characteristic peaks. Each of these peaks is associated with key developmental transitions and prepares the larva for metamorphosis. Low nutrition delays attainment of critical size and the initiation of these peaks, but also appears to 
raise basal levels of circulating ecdysone between these peaks ([74], Supplementary Figure 1), which slows the growth of the body [74]. At the same time, low nutrition also lowers levels of circulating insulin-like peptides, further slowing growth of the body. Counterintuitively, however, low nutrition appears to have opposing effects on imaginal disc growth, suppressing growth by lowering insulin-signalling, but promoting growth, at least during the middle of the third instar, by increasing ecdysone signalling.

Our data suggest that these opposing effects are critical to robust patterning of the wing under different nutritional conditions. At low nutritional conditions, low insulin-signalling at the beginning of the third larval instar slows growth of the body and of the imaginal discs. At this stage, growth of the wing imaginal discs is largely independent of ecdysone [63], evident from the near-wildtype growth of the wing discs during this period in PGX larvae (Figure 2). In the middle of the third larval instar, however, low nutritional conditions elevate basal levels of ecdysone [74]. This drives disc growth independent of insulin-signalling to ensure the discs are of sufficient size to generate viable adult appendages, even as elevated ecdysone suppresses growth of the body as a whole $[49,64,68]$. At the same time, changes in the tempo of the ecdysone fluctuations may ensure that patterning is initiated at the appropriate developmental time, when discs are a sufficient size to generate a viable adult appendage. Three factors therefore appear necessary to achieve variable size but robust patterning under a range of nutritional conditions: (1) a graded growth response to ecdysone; (2) nutritionally-sensitive growth that is independent of ecdysone; and (3) a threshold patterning response to ecdysone.

There is some evidence that our findings apply to patterning and growth of the wings in other insect species. In the tobacco hornworm Manduca sexta and the buckeyed butterfly Junonia coenia, wing disc growth is regulated by both ecdysone and insulin $(73,76)$. Further, in J. coenia, wing vein patterning progresses independently of wing size in starved versus fed caterpillars [75]. Thus, the independent regulation of growth and patterning, with growth regulated by both insulin and ecdysone signalling, may be a general mechanism to achieve robust patterning across a range of wing sizes.

While ecdysone and insulin signalling provide systemic cues that tune organ growth to the environmental conditions, morphogens like Wingless and Decapentaplegic (Dpp) act to regulate growth in an organ autonomous manner. The extent to which morphogen gradients respond to these systemic cues is unclear, although the activity of morphogens is known to interact with those of systemic signals at the level of the target genes. For example, insulin/TOR-signalling regulates the activity of Yorkie, a downstream effector of patterning morphogens, including 
Wingless and Dpp, which controls the rate of cell division [76]. Similarly, reducing ecdysone signalling in the wing reduces the expression of Wingless and reduces Dpp signalling, measured by the levels of phosphorylated Mothers against Dpp expression [56, 58,59]. Taken together, the signalling pathways that regulate organ growth in response to environmental conditions interact in complex ways with those that regulate organ-autonomous growth suggesting that these two growth regulating mechanisms are not as independent as previously thought [6].

Although the growth of the disc relies on insulin and ecdysone signalling, the progression of patterning for Achaete and Senseless in the wing disc appears to be driven by threshold responses to ecdysone. This is not to say that the progression of patterning does not depend on environmental conditions. Indeed, starvation early in the third instar impedes patterning in both the wing and ovary of $D$. melanogaster $[59,60]$. However, rather than resulting from a direct effect of insulin signalling on patterning, the block in the progression of pattern occurs because insulin signalling controls the timing of the first ecdysone pulse in the third larval instar [52, 77]. Our results here confirm that patterning requires suprathreshold concentrations of ecdysone to be initiated. Further, the manner in which ecdysone regulates the progression of patterning ensures that it remains robust against further environmental perturbation. By switching on pattern above threshold ecdysone concentrations, the disc can continue to pattern across a range of environmental conditions, even while growth retains sensitivity to those conditions.

A similar threshold mechanism appears to regulate patterning in the wing discs of other insects. In J. coenia, the patterning stage of wing discs can be quantified by the extent of tracheal invasion into the wing pouch [75]. As for Drosophila, the earliest stages of patterning depend on nutrition in the butterfly J. coenia. If caterpillars are starved before the wing discs begin to pattern, then their discs remain small and their veins unpatterned [75]. In caterpillars starved at later stages after disc patterning has been initiated, however, the wing discs are small but reach the same vein patterning stage as those of fed control animals. Whether or not the initiation of patterning in $J$. coenia also depends on ecdysone has yet to be determined.

Our data suggest that Achaete and Senseless both show threshold responses to ecdysone, but that they differ in the threshold of ecdysone that initiates patterning. While Achaete patterns advance to stage four between 0 and 15 hours after the moult to the third instar, Senseless only progresses to stage two at 15 hours [62]. Given that Achaete initiates progression at 5 hours after the moult, while ecdysone titres are just beginning to increase [52], it seems likely that Achaete requires less ecdysone to initiate patterning progression. This hypothesis is supported by the observation that feeding larvae $12.5 \mathrm{ng} / \mathrm{ml}$ of ecdysone is more-or-less sufficient to 
initiate Achaete patterning (albeit incompletely in starved larvae), but insufficient to initiate Senseless patterning. This difference in threshold might also explain why Achaete patterning is able to progress somewhat in PGX larvae. While genetically ablating the PG eliminates the ability of the animal to produce ecdysone [56], we expect that it takes some time for the gland to die after larvae are shifted to the permissive temperature. In this time, the gland might already produce sufficient ecdysone to induce some progression in Achaete patterning, but not enough to induce Senseless pattern.

The observation that patterning shows a threshold response to ecdysone may not be surprising, given that we assayed patterning through the expression of neurogenic genes, which are either detected or not detected as a bimodal response. However, our patterning scheme shows that patterning is a continuous process that, like growth, can vary in its rate of progression depending on environmental and hormonal conditions. Consequently, the progression of patterning could, in principle, also show a graded response to ecdysone levels, which we would be able to detect. Both Achaete and Senseless patterning progressed at the same rate regardless of the level of supplemental ecdysone in PGX larvae, however. Indeed, the temporal progression for both Achaete and Senseless was similar to how these patterning stages appear in control discs. The observation that the rate of patterning is independent of ecdysone level is confirmation of an assumption that, hitherto, has not been tested.

What determines the rate of pattering once it has been initiated is unclear. We did not observe discs progressing from stage 1 immediately to stage 7 within a single 5-hour time interval even at the highest ecdysone concentrations. This suggests that there are additional temporal factors that regulate how patterning progression unfolds. Almost certainly, interactions between the gene regulatory networks that regulate patterning control how patterning progresses across regions of the wing disc. We have very little understanding if/how the different regions of the wing communicate with each other to achieve this. In principle, differences between when cells turn on Achaete and Senseless across the disc could arise in response to other developmental signals, such as from the Dpp, Wingless, or Hedgehog morphogen gradients responsible for correctly scaling and patterning the wing.

Part of this temporal signature might arise from ecdysone itself. In this study we exposed animals to tonic concentrations of ecdysone. Developing larvae, however, secrete four pulses of ecdysone between the moult to third instar and pupariation [69]. We have little understanding of how developmental information is encoded within these pulses. In principle, individual pulses could either prime tissues to become responsive to hormones, or could alter their sensitivity - as the early 
ecdysone pulse does for wing disc growth and patterning $[59,60,63]$. Future studies comparing the difference between tonic and phasic exposure to hormone would help clarify the roles of the ecdysone pulses.

Temporal dynamics have been used extensively to understand how patterning remains robust to environmental conditions in other developmental contexts, particularly embryogenesis [78,79]. The stages of embryogenesis in species of $D$. melanogaster are both spatially and temporally robust across thermal environments $[78,79]$. In other words, even though lowering the temperature increases the total time it takes for embryos to develop until hatching, the proportion of total development time that they spend to reach each stage is the same. If temperature is switched from cool-to-hot or hot-to-cool early in embryogenesis, embryos show an initial increase in variation in the timing between stages, after which timing returns to its normal trajectory [79]. This means that embryos that are developing too fast or too slow will adjust their developmental rates to achieve temporal robustness.

In contrast to the remarkable invariance of embryonic development to changes in temperature, the patterning of wing discs in $D$. melanogaster larvae shows considerable variation across thermal conditions [62]. In this case, rather than carefully adjusting rates of patterning with the increase in developmental time, wing discs ensure correct patterning by aligning patterning at developmental milestones [62]. Patterning stages show significant variation across thermal conditions throughout most of the third instar, but overlap completely at the moult to third instar and at pupariation [62]. The contrast between tight temporal regulation in the embryo and loose temporal regulation with alignment at milestones in the wing discs of larvae highlights how developmental context matters, and might hint at differences in the mechanisms through which temporal regulation is achieved.

While our study has focussed on contrasting the robustness of patterning with plasticity of growth, depending on what is being measured there are instances where we expect patterning to also show plasticity [80]. For example, although the specification of cell types in the correct location within an organ may show little variation across environmental conditions, the number of structures specified can vary. The total number of abdominal and sternopleural bristles varies with temperature [81, 82], as does the number of terminal filament stacks that are specified in the ovary, which is also affected by nutrition [83-86]. Plasticity in the number of bristle cells or terminal filament stacks presumably occurs because the mechanisms that specify the number of each structure do not scale with tissues size. In other cases, the location of specific cell types may also be plastic. For example, there is an extensive literature exploring how the relative positions of veins in the wings of $D$. melanogaster and other insects is affected by environmental factors such 
as nutrition and temperature (e.g. [87-90]). Plasticity in wing shape is likely to be more complex, and may involve process that act at many different points during wing development $[91,92]$. Future studies targeting how the mechanisms that establish the position of cell types differ from those that determine the number of cells of a given type would allow us to further define what makes traits either sensitive or robust towards changes in environmental conditions, and at what level.

\section{Materials and Methods}

\section{Fly stocks and rearing conditions}

We initially manipulated growth rates and developmental timing by either altering the rearing temperature or the rates of ecdysone synthesis in developing $D$. melanogaster larvae. We used the isogenic strain, Samarkand (SAM), which also bears the $\mathrm{w}^{1118}$ mutation, when rearing larvae under one of three thermal conditions: $18^{\circ} \mathrm{C}$, $25^{\circ} \mathrm{C}$, and $29^{\circ} \mathrm{C}$. To accelerate the rates of ecdysone synthesis, we used the progeny from $w^{1118}$; phantom-GAL4, which is expressed in the prothoracic glands, crossed with yw flp; UAS InR29.4 (phm > InR). We decreased rates of ecdysone synthesis by crossing P0206-GAL4, which drives expression throughout the ring gland, with yw; UAS PTEN (P0206 > PTEN). Even though P0206-GAL4 is a weaker GAL4 driver for the prothoracic gland and also drives expression in the corpora allata, we chose to use it to drive UAS PTEN because phm>PTEN larvae die as first instar larvae [64]. The parental lines yw flp; UAS InR29.4 (+ > InR) and yw; UAS PTEN (+ > PTEN) were used as a reference for the phm $>I n R$ and $P 0206>$ PTEN genotypes respectively.

Flies of the above genotypes were raised from timed egg collections (2-6 hours) on cornmeal/molasses medium containing $45 \mathrm{~g}$ of molasses, $75 \mathrm{~g}$ of sucrose, $70 \mathrm{~g}$ of cornmeal, $10 \mathrm{~g}$ of agar, $1100 \mathrm{ml}$ of water, and $25 \mathrm{ml}$ of a $10 \%$ Nipagin solution per liter. Larvae were reared at low density (200 eggs per $60 \times 15 \mathrm{~mm}$ Petri dish) in a $12 \mathrm{~h}$ light-dark cycle with $70 \%$ humidity, and maintained at $25^{\circ} \mathrm{C}$ unless stated otherwise.

We used a transgenic combination that allowed us to genetically ablate the prothoracic gland and eliminate native ecdysone synthesis specifically in the third larval instar. We crossed a tub-GAL80ts, phantom GAL4 strain with UAS Grim to generate $P G X$ progeny (Herboso et al., 2015). GAL80 ${ }^{\text {ts }}$ is a repressor of GAL4 active at temperatures lower than $22^{\circ} \mathrm{C}$ [65]. Rearing PGX larvae at $17^{\circ} \mathrm{C}$ allows GAL80 ts to remain active, thus the phantom GAL4 cannot drive the expression of UAS grim to promote cell death. Under these conditions, larvae can moult, pupariate, and complete metamorphosis (Herboso et al., 2015). Changing the larval rearing temperature to $29^{\circ} \mathrm{C}$ disables GAL80 ts activity, thus ablating the prothoracic gland 
[56]. The progeny of the isogenic control strain, w1118, crossed with one of two parental lines, either phantom-GAL4 $(P G>+)$ or UAS Grim (+ > Grim), were used as controls for genetic background effects. The parental controls were reared under the same thermal conditions as PGX larvae.

Crosses, egg collections, and larval rearing were done on the cornmeal/molasses medium (above) for the experiments in Figure 4 or, for the experiments in Figures 5 7, on Sugar-Yeast-Agar (SYA) medium: $50 \mathrm{~g}$ of autolysed Brewer's yeast powder (MP Biomedicals), $100 \mathrm{~g}$ of sugar, $10 \mathrm{~g}$ of agar, and $1200 \mathrm{ml}$ of water. In addition, we added $3 \mathrm{ml}$ of proprionic acid and 3 grams of nipagen to the SYA medium to prevent bacterial and fungal growth. Egg collections were performed on SYA medium for 4 hours at $25^{\circ} \mathrm{C}$ or overnight at $17^{\circ} \mathrm{C}$ and larvae were reared at controlled densities of 200 eggs per food plate ( $60 \times 15 \mathrm{~mm}$ Petri dish filled with SYA medium) at $17^{\circ} \mathrm{C}$, as described previously (Herboso et al., 2015).

\section{Animal staging and developmental time}

To measure the effects of larval rearing temperature or changes in the rates in ecdysone synthesis on wing disc growth and wing disc patterning, larvae were staged into 1-hour cohorts at ecdysis to the third larval instar as in (REF). To do this, food plates were flooded with $20 \%$ sucrose and all second instar larvae were transferred to a new food plate. After one hour, the food plate was flooded once again with $20 \%$ sucrose and the newly moulted third instar larvae were collected and transferred to new food plates and left to grow until the desired time interval. Newly moulted larvae were transferred to incubators at $18^{\circ} \mathrm{C}, 25^{\circ} \mathrm{C}$ or $29^{\circ} \mathrm{C}$. Larvae reared in the $18^{\circ} \mathrm{C}$ and $29^{\circ} \mathrm{C}$ incubators were subject to constant dark due to equipment constraints. Animals were staged and their wing discs dissected at defined intervals after the larval moult as in (Oliveira et al 2014).

For the $\mathrm{PGX}, p h m>$, and $>$ Grim genotypes, larvae were raised from egg to second instar at $17^{\circ} \mathrm{C}$. Larvae were staged into 2 -hour cohorts at ecdysis to the third larval instar using the methods described above. We separated female and male larvae by examining them for the presence of testes, which are significantly larger and more visible even in newly moulted males.

\section{Exogenous ecdysone feeding treatments}

To show that ecdysone could rescue patterning and growth in PGX larvae (Fig 4C\&D), we added either $0.15 \mathrm{mg}$ of the active form of ecdysone (20hydroxyecdysone, Cayman Chemical, Item No. 16145) dissolved in ethanol, or an 
equivalent volume of ethanol, to $1 \mathrm{ml}$ of standard food. Both the ethanol- and ecdysone-supplemented food were allowed to sit at room temperature for at least 4 hours to evaporate off excess ethanol before use. Twelve larvae were transferred to one of the two supplemented foods either at $0 \mathrm{~h} \mathrm{AL3E}$ and left to feed for $42 \mathrm{~h}$ or at $42 \mathrm{~h} \mathrm{AL3E}$ and left to feed for $24 \mathrm{~h}$.

To determine the relative contributions of nutrition-dependent signalling or ecdysone to growth and patterning, we fed newly moulted PGX and control larvae 1 $\mathrm{ml}$ of starvation medium (1\% sucrose with 1\% agar) supplemented with either 0.15 mg of ecdysone dissolved in ethanol, or an equivalent volume of ethanol (Fig Suppl 4). Supplemented food was left at room temperature for at least 4 hours to evaporate excess off ethanol before use. Larvae were collected at 24 h AL3E for tissue dissection.

For the ecdysone dose response experiments, we conducted an initial pilot that showed that supplementing the food with $100 \mathrm{ng}$ of ecdysone/mg food could rescue most of the Achaete and Senseless patterning in PGX wing discs. We collected newly moulted third instar larvae, separated the sexes, and then transferred them to either sucrose food ( $20 \%$ sucrose, $1 \%$ agar; starved) or SYA food (fed) at $29^{\circ} \mathrm{C}$. We fed these larvae on one of six ecdysone concentrations: $0,6.25,12.5,25,50$, or $100 \mathrm{ng}$ of ecdysone/ $\mathrm{mg}$ food. We added the same volume of ethanol to all treatments.

To quantify the relationship between the concentration of ecdysone administered and the concentration of ecdysone in the hemolymph, we allowed newly ecdysed larvae to feed on either sucrose or SYA food that had been supplemented with one of the six concentrations of ecdysone for $20 \mathrm{~h}$ at $29^{\circ} \mathrm{C}$. We then transferred them onto either sucrose food or SYA food that did not contain ecdysone but was dyed blue. They were left to feed for 2 hours until their guts were filled with blue food. This extra step was taken so that we could be sure that our hemolymph ecdysone titres were not contaminated with ecdysone from the food. Thirty - forty larvae were then weighed as a group, and transferred to 5 times their weight in volume of ice cold methanol. Larvae were homogenized and ecdysone titres were determined using a 20-Hydroxyecdysone Enzyme ImmunoAssay Kit (Cayman Chemical, Item Number 501390) as per manufacturer's instructions.

\section{Dissections and immunocytochemistry}

For each sample, 10-20 larvae were dissected on ice cold phosphate-buffered saline (PBS) and fixed in $4 \%$ formaldehyde in PBS overnight at $4^{\circ} \mathrm{C}$. After fixation, the tissue was washed four times (15 minutes per wash) with 0.3\% Triton X-100 in PBS 
(PBT), then blocked for 30 minutes at room temperature in $2 \%$ heat-inactivated normal donkey serum in PBT. After blocking, the tissue was incubated in a primary antibody solution diluted with $2 \%$ heat-inactivated normal donkey serum in PBT overnight at $4^{\circ} \mathrm{C}$. We used the guinea pig anti-Senseless (Nolo et al., 2000, 1:1000) and mouse anti-Achaete (supernatant, 1:10) primary antibodies. To compare signal across tissues, we stained for both antigens simultaneously. The washing and blocking procedure was repeated after primary antibody incubation and then the tissue was incubated in a secondary antibody (1:200 each of anti-guinea pig (546nm) and anti-mouse $(488 \mathrm{~nm})$ ) overnight at $4^{\circ} \mathrm{C}$. The tissues were washed with PBT and rinsed with PBS and then the wing imaginal discs were mounted on poly-L-lysinecoated coverslips using Fluoromount-G (SouthernBiotech). Tissues were imaged using either a Leica LSM 510 or a Nikon C1 upright confocal microscope and processed using ImageJ (version 2.0) and Adobe Photoshop CC 2017.

\section{Quantifications of wing imaginal disc size and Achaete and Senseless pattern}

We quantified wing disc size using disc area as a proxy. All quantifications were done using ImageJ. Wing discs show exponential growth in the third instar. Thus, we studied the growth trajectories of the discs by In-transforming disc area.

Achaete and Senseless stage was quantified using the staging scheme developed by [62], associating each of the wing imaginal discs to an Achaete or Senseless stage varying from 1 to 7 .

\section{Statistical analysis}

All the analyses were conducted in $\mathrm{R}$ and the annotated $\mathrm{R}$ markdown scripts and data for the analyses are deposited on Figshare.

For the relationship between age and disc size $\left(\log \mu \mathrm{m}^{2}\right.$ ) or disc pattern (Achaete or Senseless) we fit either linear or Gompertz models and selected the model that best fit the data, using ANOVA and AIC. The Gompertz model was parameterized as $y=a e^{-b * c^{x}}$, where $y$ is disc size/pattern, $x$ is disc age, $a$ is the asymptote of $y, b$ controls where along the $\mathrm{x}$-axis the curve is positioned and $c$ is the scaling constant, such that $c=e^{-g}$ where $g$ is the growth/patterning rate (thus, the higher $g$ the lower $c$ ). To compare the parameters of linear models between treatments and genotypes we used ANOVA. To compare the parameters of Gompertz models between treatments and genotypes we used ANOVA to compared the fit of models that assign the same constants across groups versus models that assigned group-specific constants. 
For the relationship between disc size $\left(\log \mu \mathrm{m}^{2}\right)$ and Senseless pattern we fit a four parameter logistic model $y=a+\frac{(b-a)}{1+e^{(c-x) / d}}$ where $y$ is disc pattern, $a$ is the minimum asymptote, $b$ is the maximum asymptote, $c$ is the inflection point and $d$ is the scaling constant, such that $d=1 / k$, here $k$ is the logistic growth rate. We again used ANOVA to compare the fit of models that assign the same parameters across groups versus models that assigned group-specific parameters. The relationship between disc size and Achaete pattern was fit using a linear model and compared across treatments using ANOVA.

We used ANOVA to compare disc size/patten at specific time points between treatments and genotypes, using a Tukey's HSD test to allow comparison among groups.

For all parametric tests we checked for homoscedasticity and normality of errors.

\section{Acknowledgements}

We would like to thank members of the Mirth and Shingleton labs, past and present, for their useful discussions relating to this project. This research was supported by NSF grants IOS-0919855 and IOS-1557638 to AWS and an ARC Future Fellowship (FT170100259) to CKM.

\section{Competing Interests}

The authors declare that they have no competing interests. 


\section{References}

1. Nylin S, Gotthard K. Plasticity in life-history traits. Annu Rev Entomol 1998, 43:63-83.

2. West-Eberhard MJ. Phenotypic plasticity and the origins of diversity. Annual Review of Ecology and Systematics 1989, 20:249-278.

3. Beldade P, Mateus ARA, Keller RA. Evolution and molecular mechanisms of adaptive developmental plasticity. Mol Ecol 2011, 20:1347-1363.

4. Koyama T, Mendes CC, Mirth CK. Mechanisms regulating nutrition-dependent developmental plasticity through organ-specific effects in insects. Front Physiol 2013, 4:1-12.

5. Shingleton AW. The regulation of organ size in Drosophila: physiology, plasticity, patterning and physical force. Organogenesis 2010, 6:76-87.

6. Mirth CK, Shingleton AW. Coordinating Development: How Do Animals Integrate Plastic and Robust Developmental Processes? Frontiers in Cell and Developmental Biology 2019, 7:8.

7. Nijhout HF, Sadre-Marandi F, Best J, Reed MC. Systems biology of phenotypic robustness and plasticity. Integrative and Comparative Biology 2017, 57:171184.

8. Félix MA, Barkoulas M. Pervasive robustness in biological systems. Nature Reviews Genetics 2015, 16:483-496.

9. Félix MA, Wagner A. Robustness and evolution: concepts, insights and challenges from a developmental model system. Heredity 2008, 100:132-140.

10. Manu SS, Spirov AV, Gursky VV, Janssens H, Kim AR, Radulescu O, VanarioAlonso CE, Sharp DH, Samsonova M, Reinitz J. Canalization of gene expression and domain shifts in the Drosophila blastoderm by dynamical attractors. PLoS Comput Biol 2009, 5:e1000303.

11. Manu SS, Spirov AV, V.V. G, Janssens H, Kim AR, Radulescu O, Vanario-Alonso CE, Sharp DH, Samsonova M, J. R. Canalization of gene expression in the Drosophila blastoderm by gap gene cross regulation. PLoS Biol 2009, 3:e1000049.

12. Clark E. Dynamic patterning by the Drosophila pair-rule network reconciles long-germ and short-germ segmentation. PLOS Biology 2017, 15(9):e2002439. 
13. Clark E, Akam M. Odd-paired controls frequency doubling in Drosophila segmentation by altering the pair-rule gene regulatory network. eLife 2016, 5:e18215.

14. Clark E, Peel AD. Evidence for the temporal regulation of insect segmentation by a conserved sequence of transcription factors. Development 2018, 145(10):dev155580.

15. Crombach A, Wotton KR, Jiménez-Guri E, Jaeger J. Gap gene regulatory dynamics evolve along a genotype network. M. Molecular Biology and Evolution 2016, 33:1293-1307.

16. Verd B, Clark E, Wotton KR, Janssens H, Jiménez-Guri E, Crombach A, Jaeger J. A damped oscillator imposes temporal order on posterior gap gene expression in Drosophila. PLOS Biology 2018, 16(2):e2003174.

17. Wotton KR, Jiménez-Guri E, Crombach A, Janssens H, Alcaine-Colet A, Lemke S, Schmidt-Ott U, Jaeger J. Quantitative system drift compensates for altered maternal inputs to the gap gene network of the scuttle fly Megaselia abdita. elife 2015, 4:e04785.

18. Almuedo-Castillo M, Blassle A, Morsdorf D, Marcon L, Soh GH, Rogers KW, Schier AF, Muller P. Scale-invariant patterning by size-dependent inhibition of Nodal signalling. Nat Cell Biol 2018, 20:1032-1042.

19. Zhou S, Lo W-C, Suhalim JL, Digman MA, Gratton E, Nie Q, Lander AD. Free extracellular diffusion creates the Dpp morphogen gradient of the Drosophila wing disc. Current Biology 2012, 22:668-675.

20. Zhu Y, Qiu Y, Chen W, Nie Q, Lander AD. Scaling a Dpp Morphogen Gradient through Feedback Control of Receptors and Co-receptors. Dev Cell 2020, 53(6):724-739.e714.

21. Schwank G, Tauriello G, Yagi R, Kranz E, Koumoutsakos P, Basler K. Antagonistic growth regulation by Dpp and Fat drives uniform cell proliferation. Dev Cell 2011, 20:123-130.

22. Wartlick O, Mumcu P, Kicheva A, Bittig T, Seum C, Jülicher F, González-Gaitán M. Dynamics of Dpp signaling and proliferation control. Science 2011, 331:1154-1159.

23. Hamaratoglu F, de Lachapelle AM, Pyrowolakis G, Bergmann S, Affolter M. Dpp Signaling Activity Requires Pentagone to Scale with Tissue Size in the Growing Drosophila Wing Imaginal Disc. PLOS Biology 2011, 9(10):e1001182. 
24. Corson F, Couturier L, Rouault H, Mazouni K, Schweisguth F. Self-organized Notch dynamics generate stereotyped sensory organ patterns in Drosophila. Science 2017, 356:eaai7407.

25. Raspopovic J, Marcon L, Russo L, Sharpe J. Modeling digits. Digit patterning is controlled by a Bmp-Sox9-Wnt Turing network modulated by morphogen gradients. Science 2014, 345:566-570.

26. Dubrulle J, McGrew MJ, Pourquié O. FGF signalling controls somite boundary position and regulates segmentation clock control of spatiotem- poral Hox gene activation. Cell 2001, 106:219-232.

27. Bakera RE, Schnella S, Maini PK. A clock and wavefront mechanism for somite formation Develomental Biology 2006, 293:116-126.

28. Nijhout HF. The control of body size in insects. Dev Biol 2003, 261:1-9.

29. Nijhout HJ, Riddiford LM, Mirth CK, Shingleton AW, Suzuki Y, Callier V. The developmental control of size in insects. WIREs Developmental Biology 2014, 3:113-134.

30. Mirth CK, Shingleton AW. Integrating body and organ size in Drosophila: recent advances and outstanding problems. Front Endocrinol 2012, 3:1-13.

31. Azevedo RB, French V, Partridge L. Temperature modulates epidermal cell size in Drosophila melanogaster. Journal of Insect Physiology 2002, 48:231-237.

32. David JR, Moreteau B, Gauthier JP, Pétavy G, Stockel A, et al. Reaction norms of size characters in relation to growth temperature in Drosophila melanogaster: an isofemale lines analysis. . Genet Sel Evol 1994, 26:229-251.

33. French V, Feast M, Partridge L. Body size and cell size in Drosophila: the developmental response to temperature. Journal of Insect Physiology 1998, 44:1081-1089.

34. James AC, Azevedo RB, Partridge L. Genetic and environmental responses to temperature of Drosophila melanogaster from a latitudinal cline. Genetics 1997, 146:881-890.

35. Partridge L, Barrie B, Fowler K, French V. Evolution and development of body size and cell size in Drosophila melanogaster in response to temperature. Evolution 1994, 48:1269-1276.

36. Grunert LW, Clarke JW, Ahuja C, Eswaran H, Nijhout HF. A quantitative analysis of growth and size regulation in Manduca sexta: the physiological basis of variation in size and age at metamorphosis. PLOS ONE 2015, 10(5):e01279880127923. 
37. Reynolds S, Notthingham SF. Effects of temperature on growth and efficiency of food utilization in fifth-instar caterpillars of the tobacco hornworm, Manduca sexta. Journal of Insect Physiology 1985, 31:129-134.

38. Thomas RH. Ecology of body size in Drosophila buzzatii: untangling the effects of temperature and nutrition. Ecol Ent 1993, 18:84-90.

39. Callier V, Nijhout HF. Control of body size by oxygen supply reveals sizedependent and size-independent mechanisms of molting and metamorphosis. Proc Nat Aca Sci USA 2011, 108:14664-14669.

40. Callier V, Shingleton AW, Brent CS, Ghosh SM, Kim J, Harrison JF. The role of reduced oxygen in the developmental physiology of growth and metamorphosis initiation in Drosophila melanogaster. J Exp Biol 2013, 216:4334-4340.

41. Glendinning Jl. How do herbivorous insects cope with noxious secondary plant compounds in their diet? Entomologia Experimentalis et Applicata 2002, 104(1):15-25.

42. Wu Q, Brown MR. Signaling and function of insulin-like peptides in insects. Annu Rev Entomol 2005, 51(1):1-24.

43. Brogiolo W, Stocker H, Ikeya T, Rintelen F, Fernandez R, et al. An evolutionarily conserved function of the Drosophila insulin receptor and insulin-like peptides in growth control. Curr Biol 2001, 11:213-221.

44. Ikeya T, Galic M, Belawat P, Nairz K, Hafen E. Nutrient-dependent expression of insulin-like peptides from neuroendocrine cells in the CNS contributes to growth regulation in Drosophila. Curr Biol 2002, 12:1293-1300.

45. Chen C, Jack J, Garofalo RS. The Drosophila insulin receptor is required for normal growth. Endocrinology 1996, 137:846-856.

46. Yenush L, Fernandez R, Myers MGJ, Grammer TC, Sun XJ, et al. The Drosophila insulin receptor activates multiple signaling pathways but requires insulin receptor substrate proteins for DNA synthesis. Mole Cell Biol 1996, 16:25092517.

47. Géminard C, Rulifson EJ, Léopold P. Remote control of insulin secretion by fat cells in Drosophila. Cell Metabolism 2009, 10(3):199-207.

48. Weinkove D, Leevers SJ. The genetic control of organ growth: insights from Drosophila. Current Opinion in Genetics \& Development 2000, 10(1):75-80. 
49. Caldwell PE, Walkiewicz M, Stern M. Ras activity in the Drosophila prothoracic gland regulates body size and developmental rate via ecdysone release. Curr Biol 2005, 15:1785-1795.

50. Colombani J, Andersen DS, Leopold P. Secreted peptide Dilp8 coordinates Drosophila tissue growth with developmental timing. Science 2012, 336:582585.

51. Mirth CK, Truman JW, Riddiford LM. The role of the prothoracic gland in determining critical weight for metamorphosis in Drosophila melanogaster. Curr Biol 2005, 15:1796-1807.

52. Koyama T, Rodrigues MA, Athanasiadis A, Shingleton AW, Mirth CK. Nutritional control of body size through FoxO-Ultraspiracle mediated ecdysone biosynthesis. eLife 2014, 3:e03091.

53. Shingleton AW, Das J, Vinicius L, Stern DL. The temporal requirements for insulin signalling during development in Drosophila. PLoS Biol 2005, 3:e289.

54. Ghosh SM, Testa ND, Shingleton AW. Temperature-size rule is mediated by thermal plasticity of critical size in Drosophila melanogaster. Proc R Soc B 2013, 280:20130174.

55. Stieper BC, Kupershtok M, Driscoll MV, Shingleton AW. Imaginal discs regulate developmental timing in Drosophila melanogaster. Dev Biol 2008, 321(1):1826.

56. Herboso L, Oliveira MM, Talamillo A, Pérez C, González M, Martín D, Sutherland JD, Shingleton AW, Mirth CK, Barrio R. Ecdysone promotes growth of imaginal discs through the regulation of Thor in $D$. melanogaster. Scientific Reports 2015, 5:12383.

57. Gokhale RH, Hayashi T, Mirque CD, Shingleton AW. Intra-organ growth coordination in Drosophila is mediated by systemic ecdysone signaling. Developmental Biology 2016, 418:135-145.

58. Dye NA, Popović M, Spannl S, Etournay R, Kainmüller D, Ghosh S, Myers EW, Jülicher F, Eaton S. Cell dynamics underlying oriented growth of the Drosophila wing imaginal disc. Development 2017, 144:4406-4421.

59. Mirth CK, Truman JW, Riddiford LM. The Ecdysone Receptor controls the postcritical weight switch to nutrition-independent differentiation in Drosophila wing imaginal discs. Development 2009, 136(14):2345-2353. 
60. Mendes CM, Mirth CK. Stage-specific plasticity in ovary size is regulated by insulin/insulin-like growth factor and ecdysone signalling in Drosophila. Genetics 2016, 202:703-719.

61. Gancz D, Lengil T, Gilboa L. Coordinated regulation of niche and stem cell precursors by hormonal signaling. PLoS Biology 2011, 9:e1001202.

62. Oliveira MM, Shingleton AW, Mirth CK. Coordination of wing and whole-body development at developmental milestones ensures robustness against environmental and physiological pertubations. PLoS Genet 2014, 10:e1004408.

63. Shingleton AW, Mirth CK, Bates PW. Developmental model of static allometry in holometabolous insects. Proc R Soc B 2008, 275:1875-1885.

64. Mirth C, Truman JW, Riddiford LM. The role of the prothoracic gland in determining critical weight for metamorphosis in Drosophila melanogaster. Current biology: CB 2005, 15(20):1796-1807.

65. McGuire SE, Le PT, Osborn AJ, Matsumoto K, Davis RL. Spatiotemporal rescue of memory dysfunction in Drosophila. Science 2003, 302:1765-1768.

66. McGuire SE, Mao Z, Davis RL. Spatiotemporal gene expression targeting with the TARGET and gene-switch systems in Drosophila. Science Signalling 2004, 2004:16.

67. Nijhout HF, Wheeler DE. Growth models of complex allometries in holometabolous insects. The American Naturalist 1996, 148:40-56.

68. Colombani J, Bianchini L, Layalle S, Pondeville E, Dauphin-Villemant C, Antoniewski C, Carre C, Noselli S, Leopold P. Antagonistic actions of ecdysone and insulins determine final size in Drosophila. Science 2005, 310(5748):667670.

69. Warren JT, Yerushalmi Y, Shimell MJ, O'Connor MB, Restifo LL, Gilbert LI. Discrete pulses of molting hormone, 20-hydroxyecdysone, during late larval development of Drosophila melanogaster: correlations with changes in gene activity. Dev Dyn 2006, 235:315-326.

70. Riddiford LM. Hormones and Drosophila Development. In: The Development of Drosophila melanogaster. Edited by Bates M, Martinez-Arias A, vol. 2. Plainview: Cold Spring Harbor Laboratory Press; 1993: 899-939.

71. Mirth CK, Tang HY, Makohon-Moore SC, Salhadar S, Gokhale RH, Warner RD, Koyama T, Riddiford LM, Shingleton AW. Juvenile hormone regulates body 
size and perturbs insulin signaling in Drosophila. Proc Natl Acad Sci U S A 2014, 111:7018-7023.

72. Parker NF, Shingleton AW. The coordination of growth among Drosophila organs in response to localized growth-perturbation. Dev Biol 2011, 357:318325.

73. Testa ND, Ghosh SM, Shingleton AW. Sex-specific weight loss mediates sexual size dimorphism in Drosophila melanogaster. PLoS One 2013, 8:e58936.

74. Lee GJ, Han G, Yun HM, Lim JJ, Noh S, Lee J, Hyun S. Steroid signaling mediates nutritional regulation of juvenile body growth via IGF-binding protein in \&lt;em\&gt;Drosophila\&lt;/em\&gt. Proceedings of the National Academy of Sciences 2018, 115(23):5992.

75. Miner AL, Rosenberg AJ, Nijhout HF. Control of growth and differentiation of the wing imaginal disk of Precis coenia (Lepidoptera: Nymphalidae). Journal of Insect Physiology 2000, 46:251-258.

76. Parker J, Struhl G. Scaling the Drosophila wing:TOR-dependent target gene access by the Hippo pathway transducer Yorkie. PLoS Biol 2015, 13:e1002274.

77. Ohhara Y, Kobayashi S, Yamanaka N. Nutrient-Dependent Endocycling in Steroidogenic Tissue Dictates Timing of Metamorphosis in Drosophila melanogaster. PLOS Genetics 2017, 13(1):e1006583.

78. Kuntz SG, Eisen MB. Drosophila embryogenesis scales uniformly across temperature in developmentally diverse species. PLoS Genet 2014, 10(4):e1004293.

79. Chong J, Amourda C, Saunders TE. Temporal development of Drosophila embryos is highly robust across a wide temperature range. Journal of the Royal Society Interface 2018, 15:20180304.

80. Mirth CK, Saunders TE, Amourda C. Growing Up in a Changing World: Environmental Regulation of Development in Insects. Annu Rev Entomol 2021, 66(1):null.

81. Moreteau B, David JR. Phenotypic plasticity and reaction norms of abdominal bristle number in Drosophila melanogaster. Journal of Bioscience 2005, 30:689-697.

82. Moreteau B, Gibert P, Delpuech J-M, Petavy G, David JR. Phenotypic plasticity of sternopleural bristle number in temperate and tropical populations of Drosophila melanogaster. Genetical Research 2003, 81:25-32. 
83. David JR. Le nombre d'ovarioles chez Drosophila melanogaster: relation avec la fécondité et valeur adaptive. Arch Zool Exp Gen 1970, 111:357-370.

84. Delpuech JM, Moreteau B, Chiche J, Pla E, Vouidibio J, David JR. Phenotypic plasticity and reaction norms in temperate and tropical populations of Drosophila melanogaster - Ovarian size and developmental temperature. Evolution 1995, 49(4):670-675.

85. Green DA, Extavour CG. Insulin signalling underlies both plasticity and divergence of a reproductive trait in Drosophila. Proceedings of the Royal Society B: Biological Sciences 2014, 281:20132673-20132673.

86. Hodin J, Riddiford LM. Different mechanisms underlie phenotypic plasticity and interspecific variation for a reproductive character in Drosophilids (Insecta: Diptera). Evolution 2000, 54(5):1638-1653.

87. Debat V, Bégin M, Legout H, David JR. Allometric and nonallometric components of Drosophila wing shape respond differently to developmental temperature. Evolution 2003, 57:2773-2784.

88. Debat V, Debelle A, Dworkin I. Plastitcity, canalization, and developmental stability of the Drosophila wing: joint effects of mutation and developmental temperature. Evolution 2009, 63(11):2864-2876.

89. Outomuro D, Adams DC, Johansson F. Wing shape allometry and aerodynamics in calopterygid damselflies: a comparative approach. BMC Evolutionary Biology 2013, 13(1):118.

90. Bitner-Mathé BC, Klaczko LB. Size and shape heritability in natural populations of Drosophila mediopunctata: temporal and microgeographical variation. Genetica 1999, 105:35-42.

91. Matamoro-Vidal A, Salazar-Ciudad I, Houle D. Making quantitative morphological variation from basic developmental processes: Where are we? The case of the Drosophila wing. Developmental Dynamics 2015, 244(9):10581073.

92. Cobham AE, Mirth CK. The development of body and organ shape. BMC Zoology 2020, 5(1):14. 
A

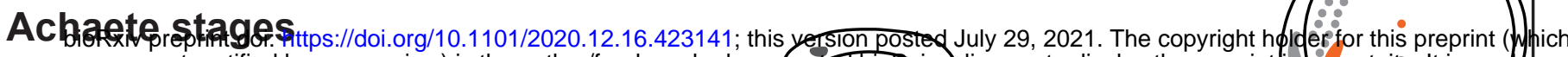
was not certified by peer review) is the author/funder, who has grantedbioRxiv a license to display the preprint in perpetuity. It is mad 8
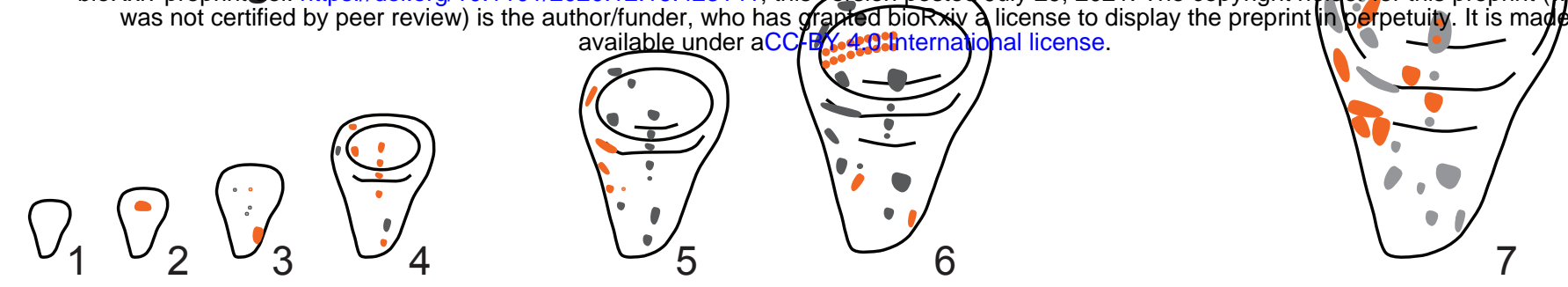

Senseless stages

B
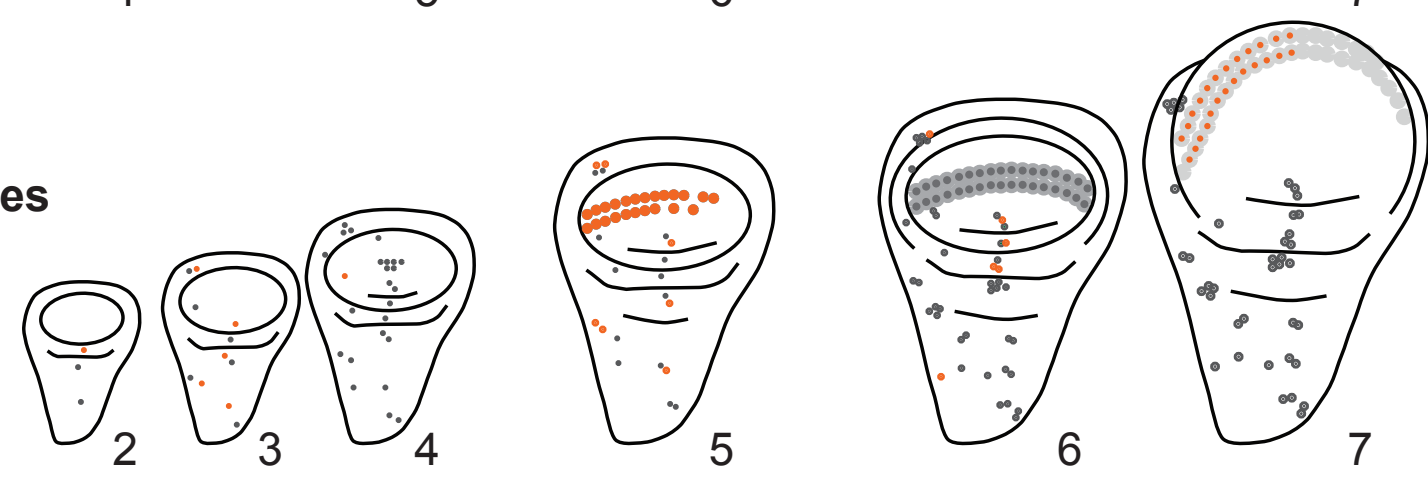

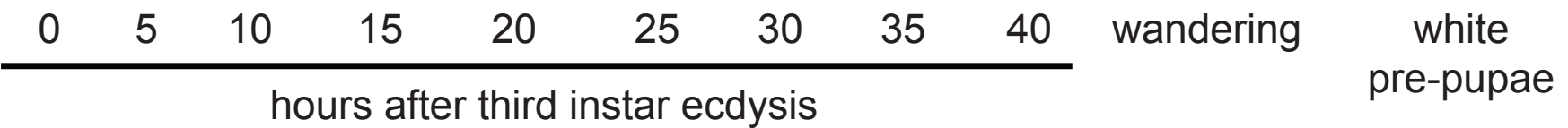

- Fast-growing disc

- Slow-growing disc

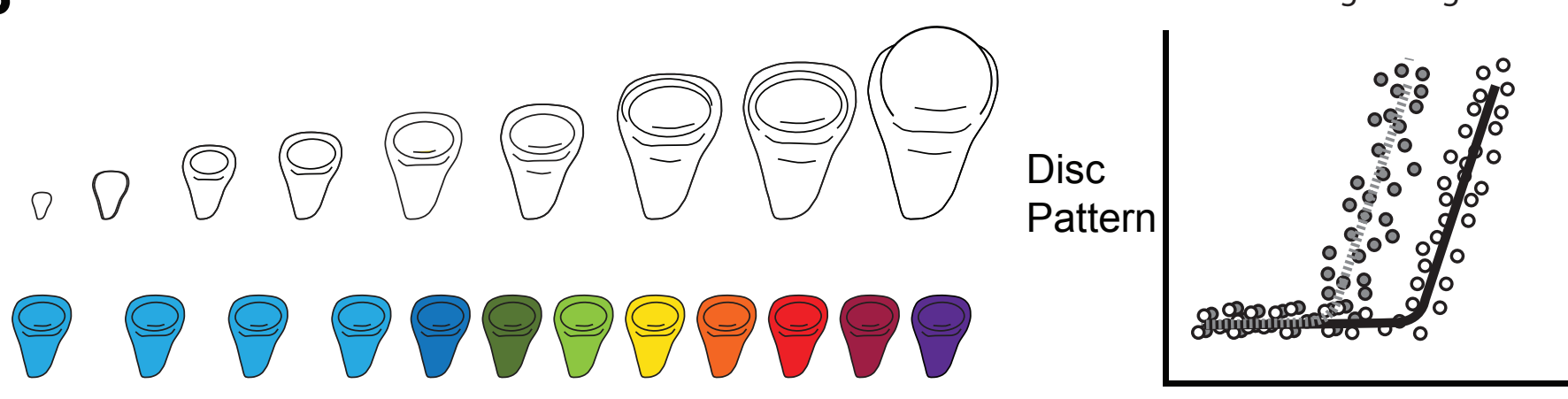

Time

C
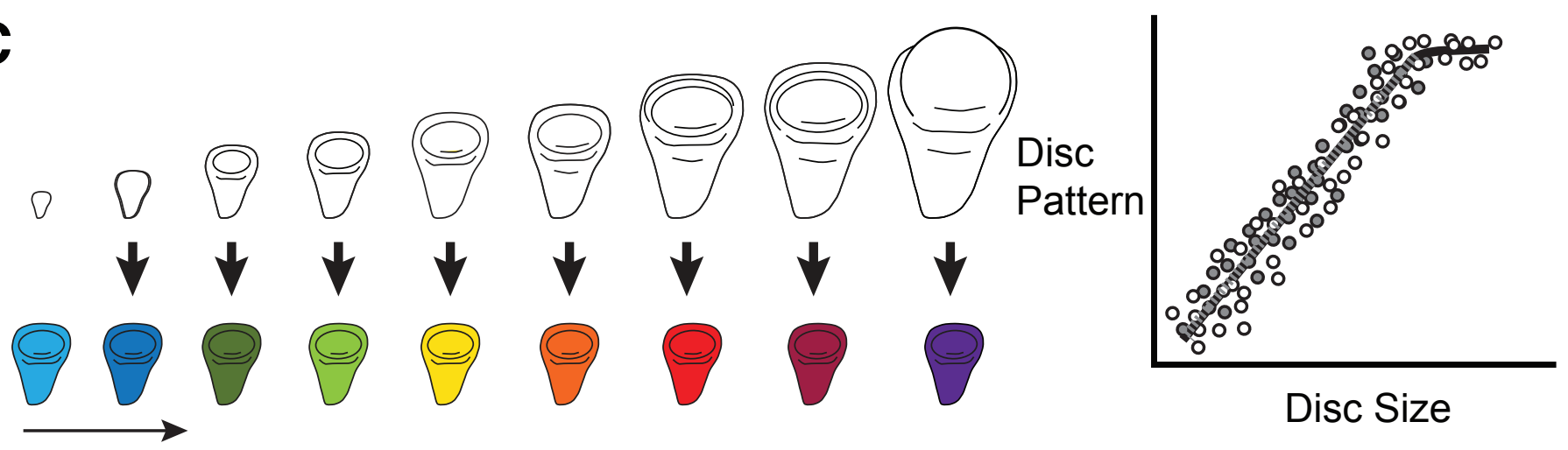

Time

D

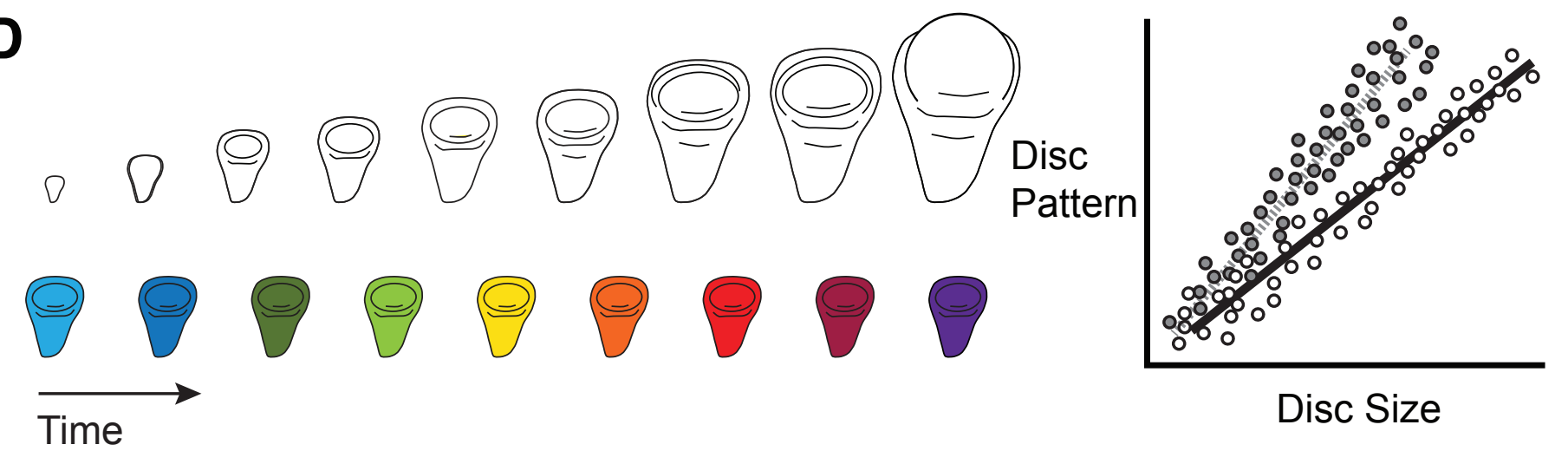




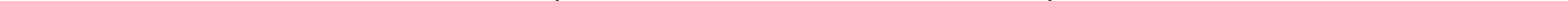




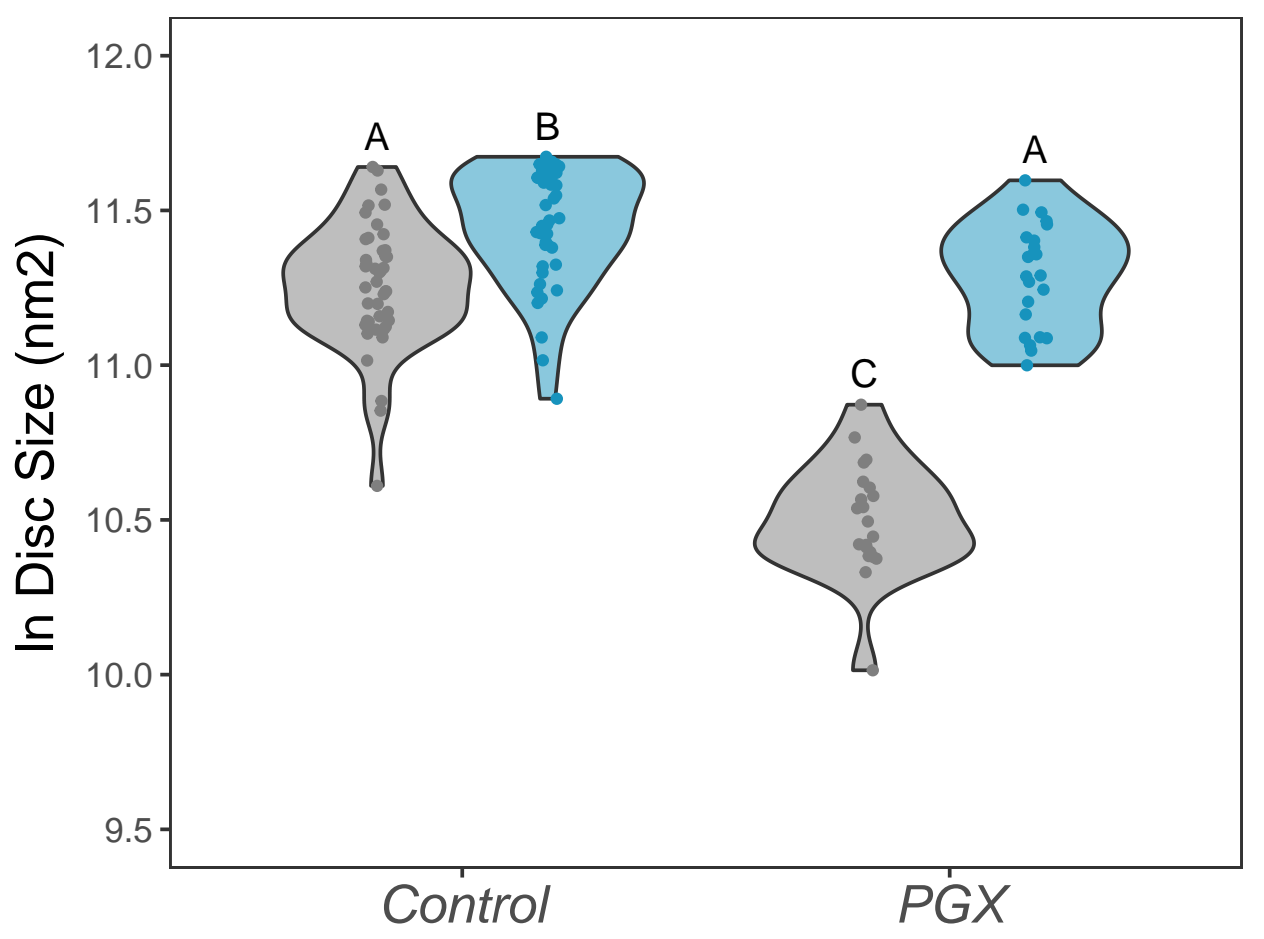

B

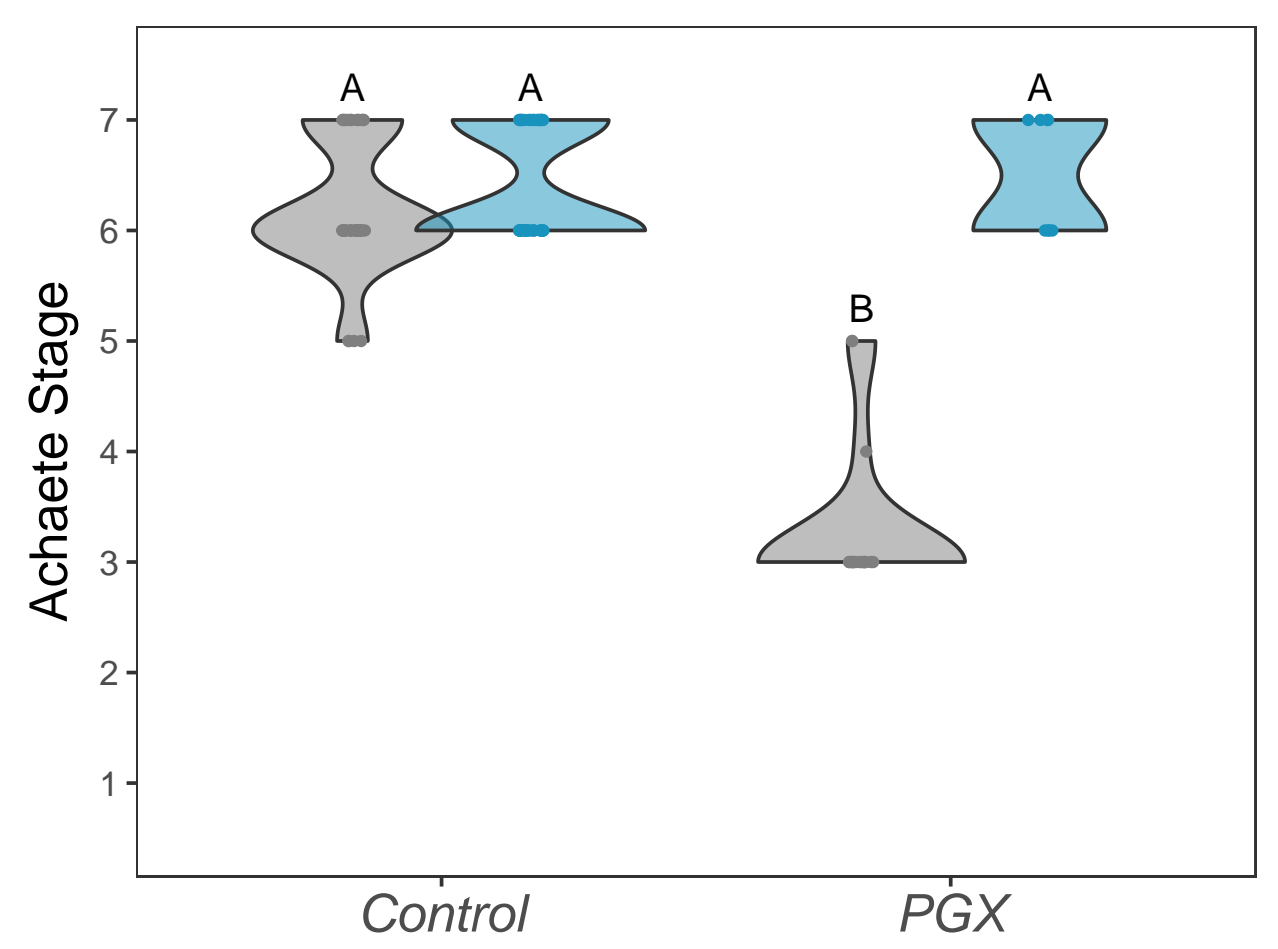

- Ethanol

Ecdysone

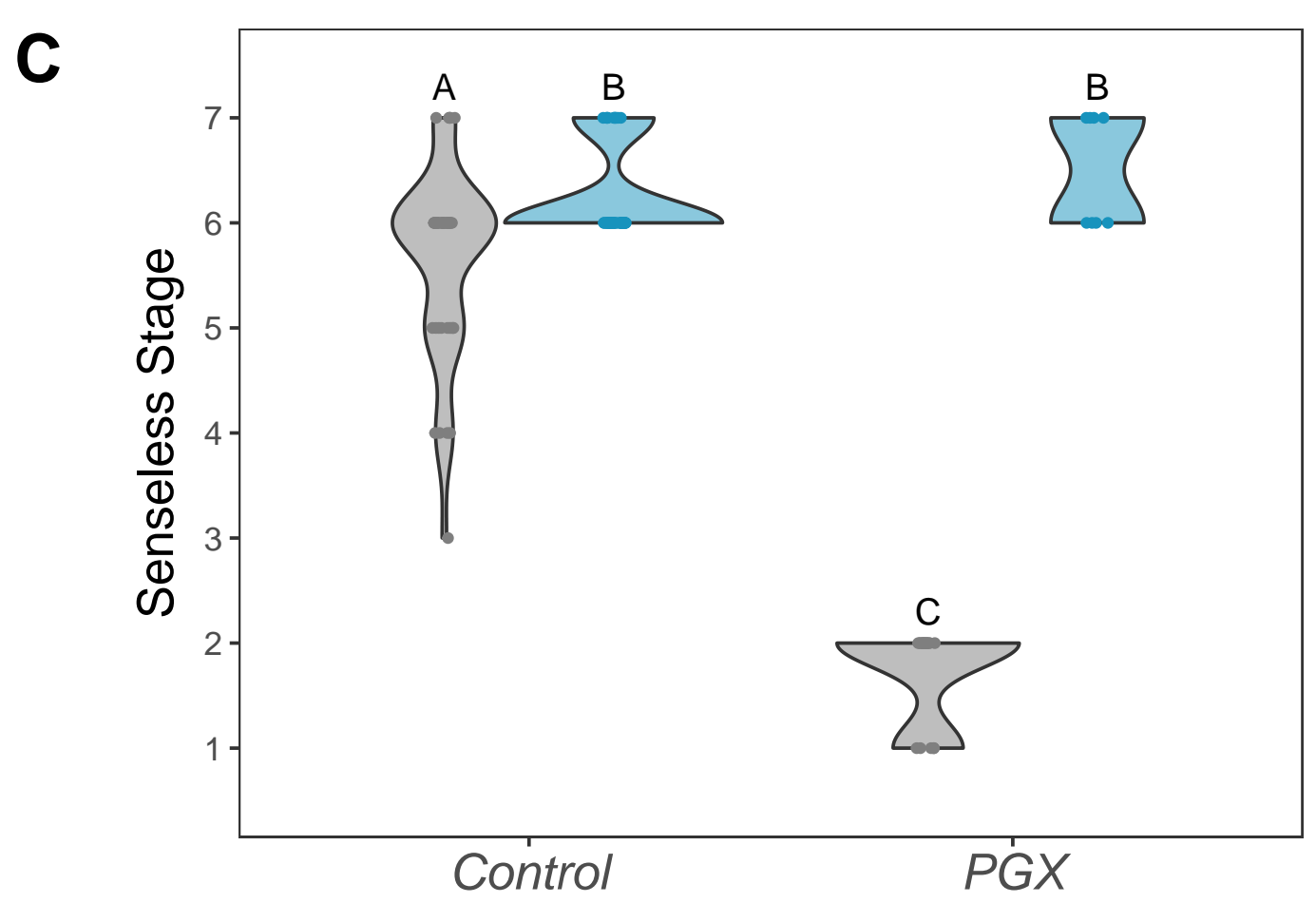


A

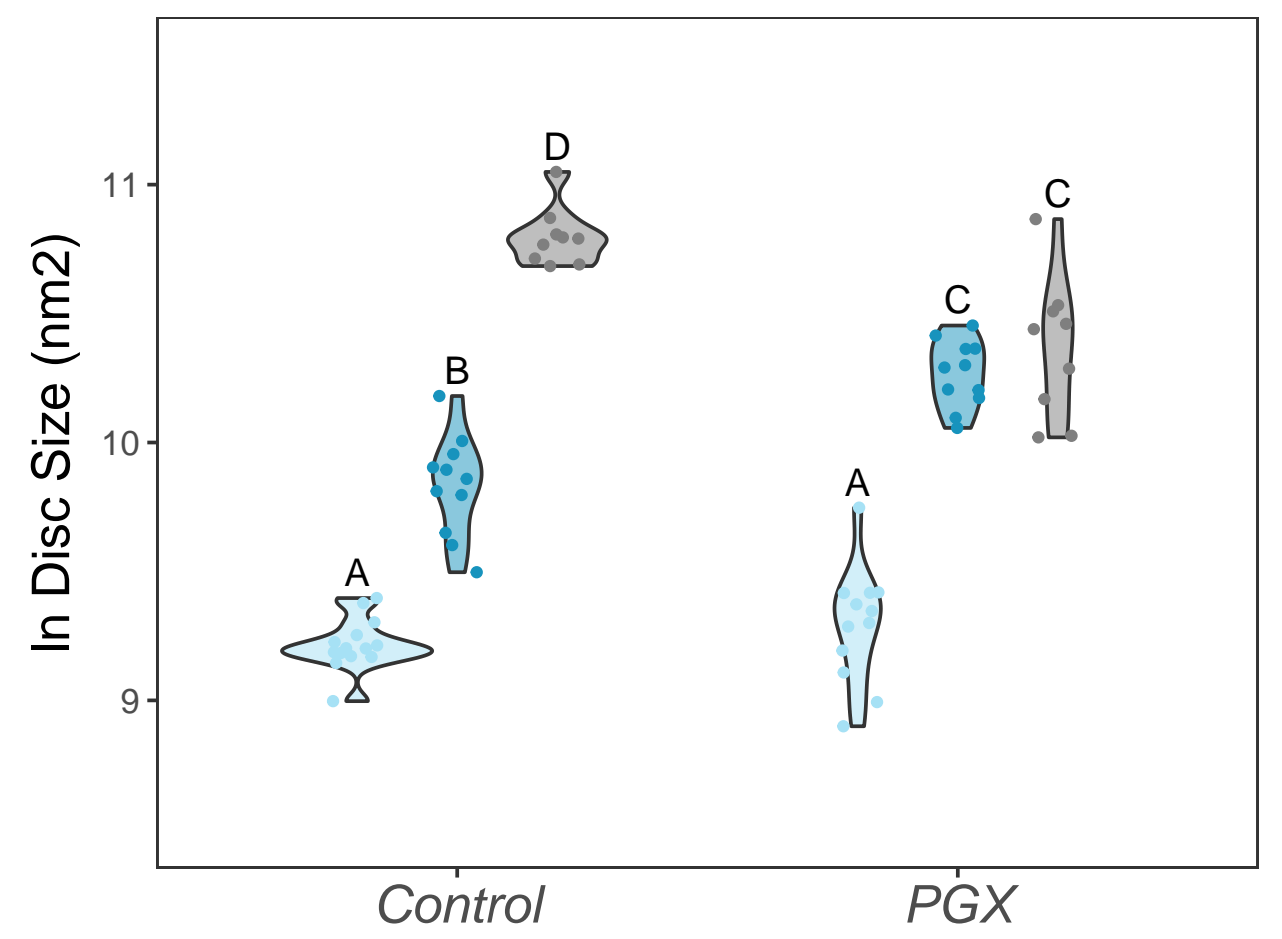

B
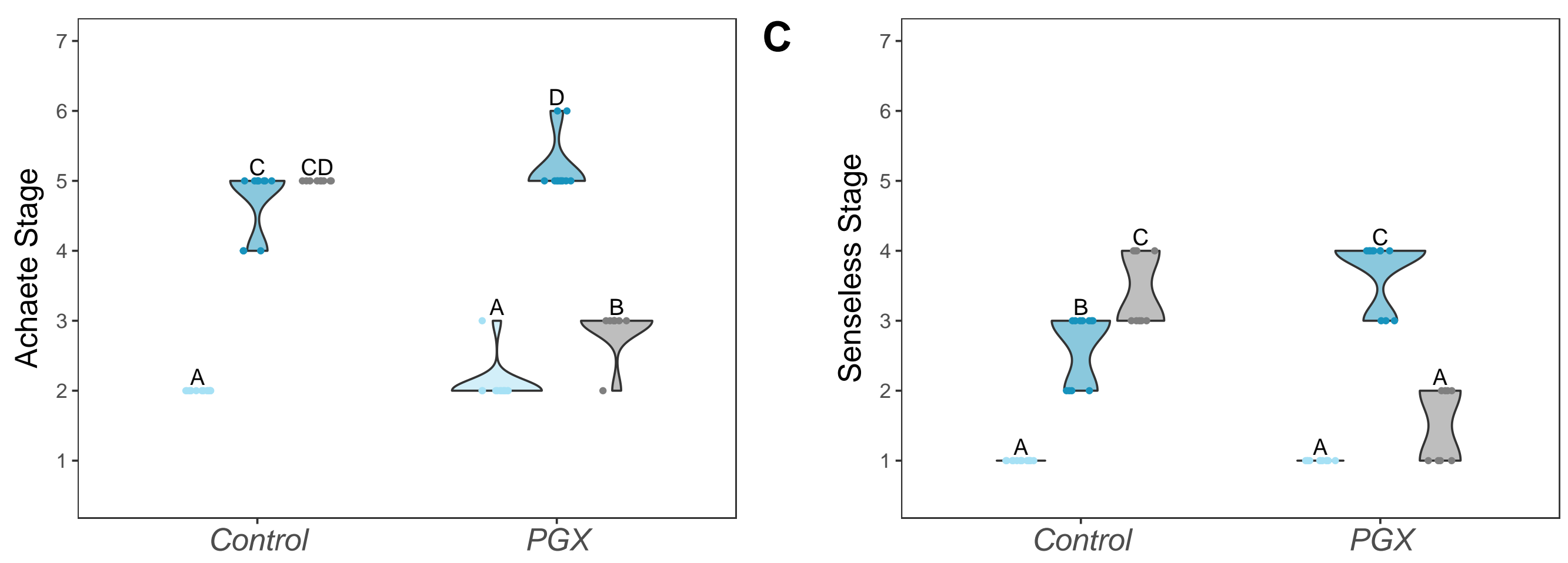
A

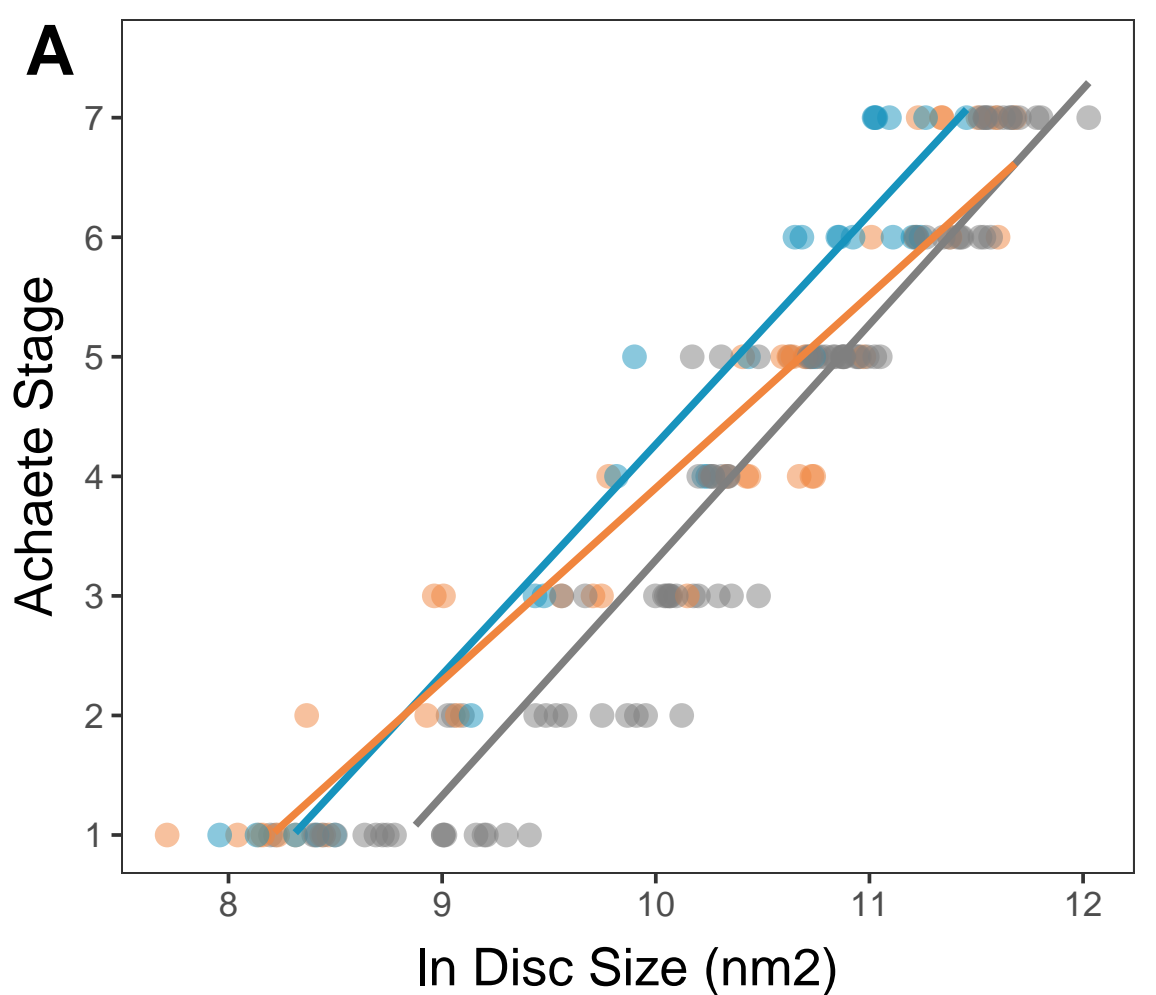

B

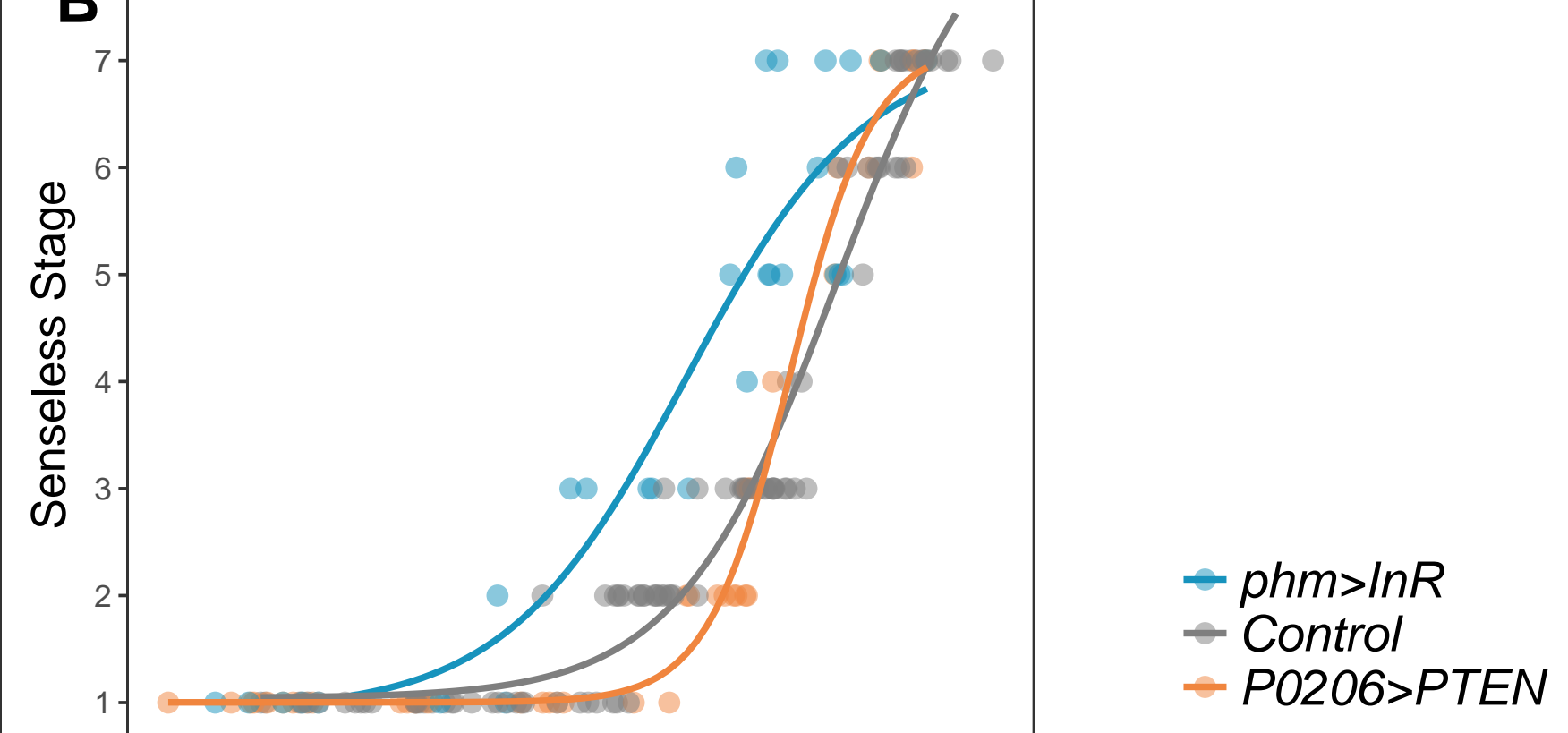




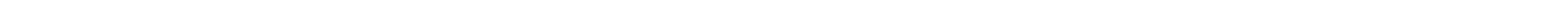


A Fed - PGX

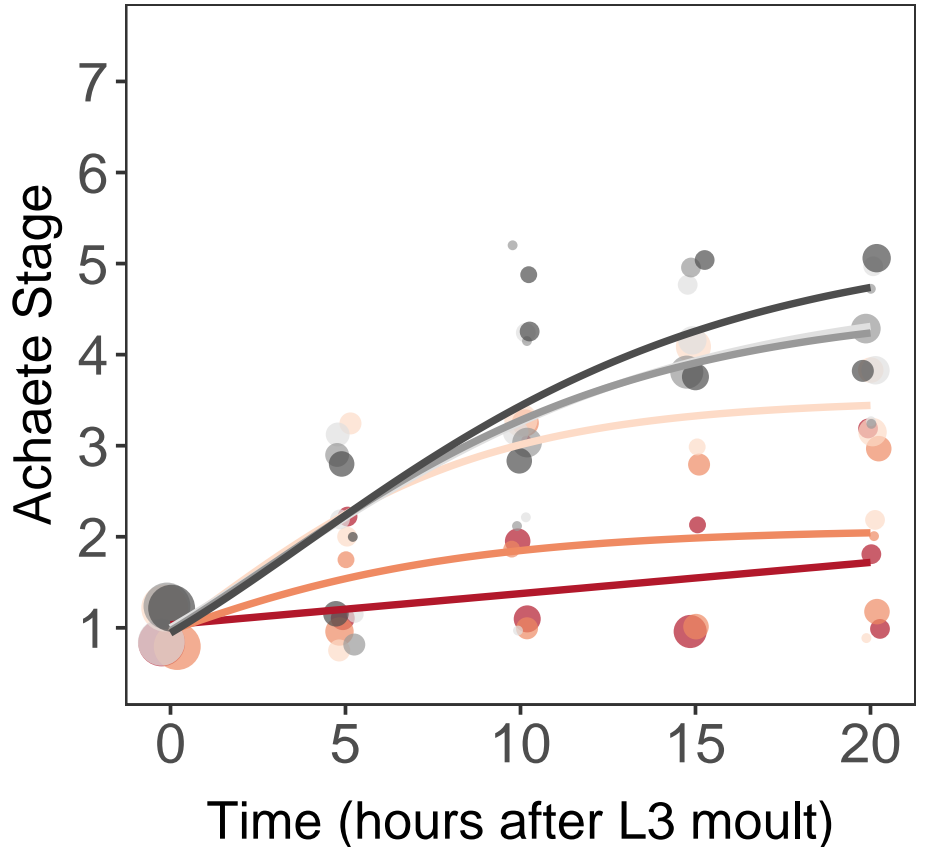

B

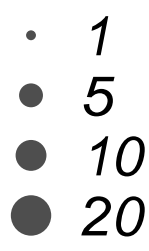

$-0-A$

$-6.25-A$ $12.5-B$ $25-C$

$-50-C$

$-100-C$

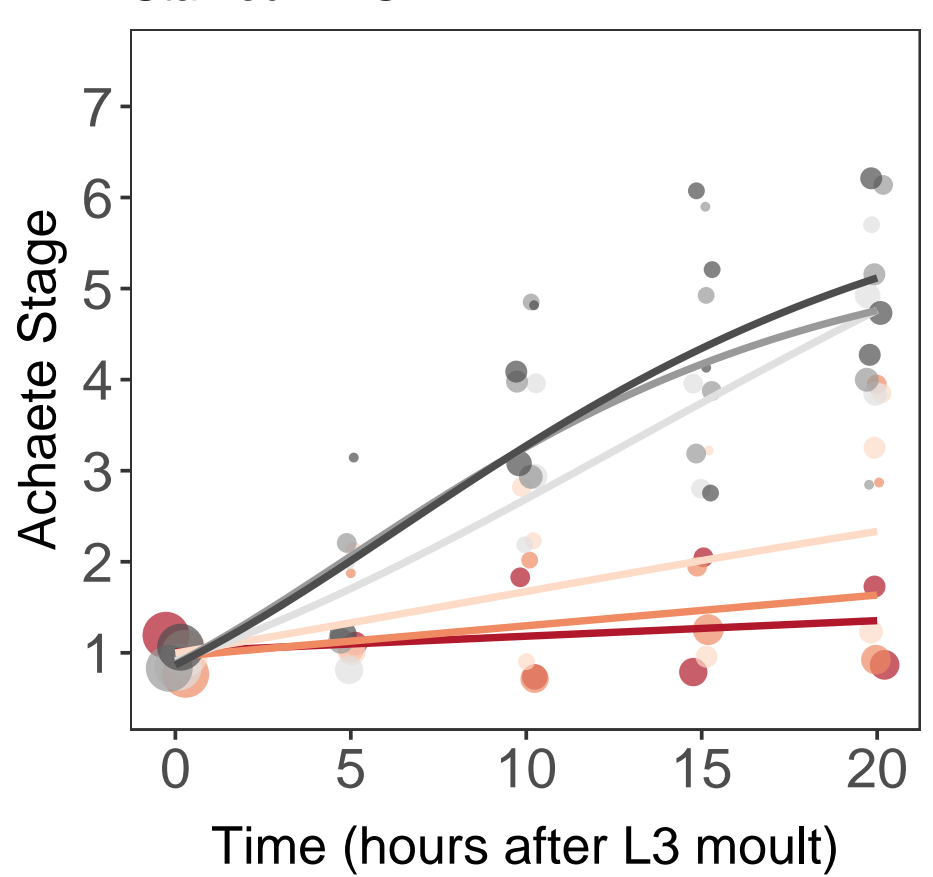

- 1

- 5

- 10

- 20

$-0-A$

$-6.25-A$ $12.5-B$ $25-C$

$-50-C$

$-100-C$ 
A Fed-PGX

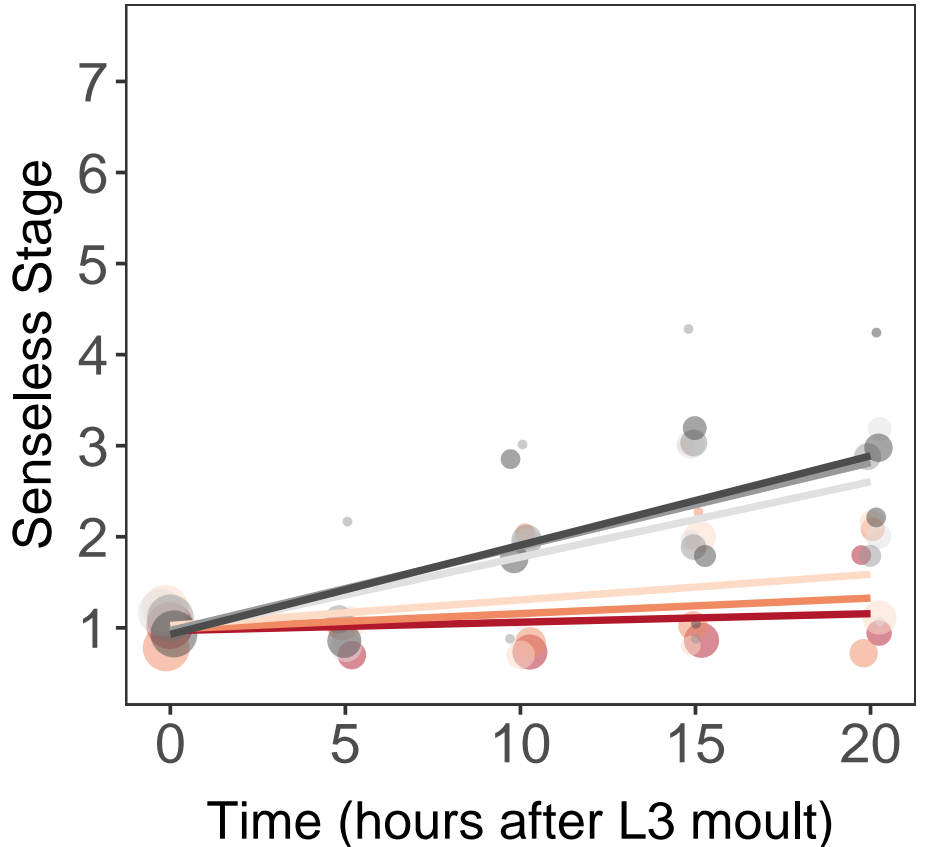

B

$-0-A$
$-6.25-A$ $12.5-A$

$-25-B$

$-50-B$

$-100-B$

- 1
-5

- 10

20

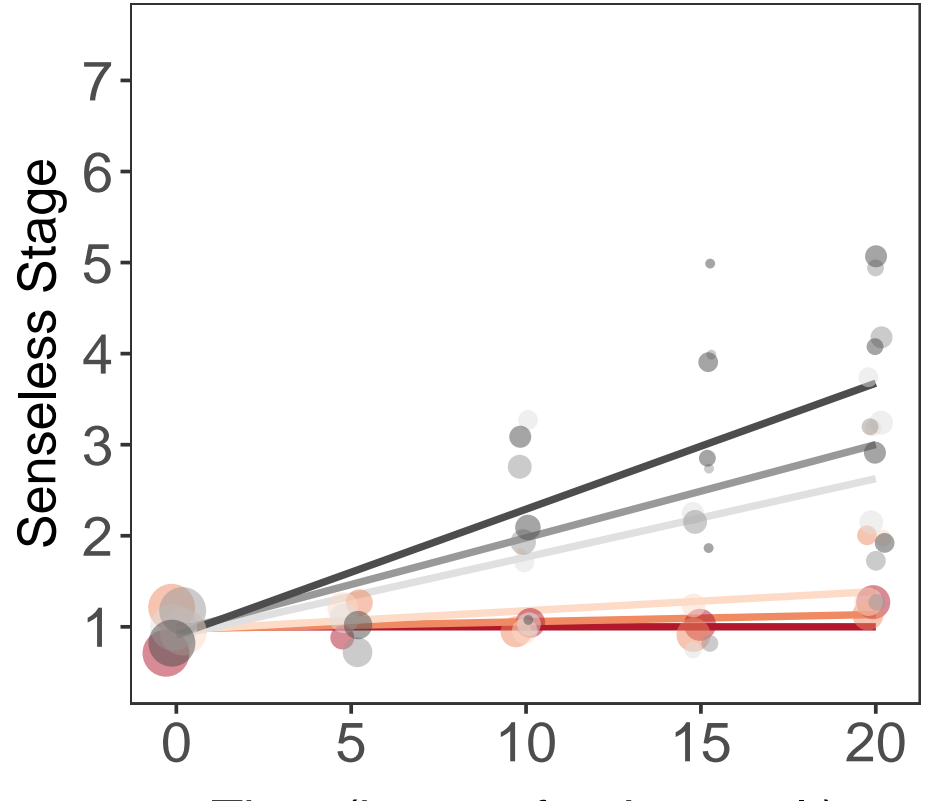

Time (hours after L3 moult)
$-0-A$

$-6.25-A$ $12.5-A$ $25-B$

$-50-B$

$-100-C$

- 1

- 5

- 10

- 20 